\title{
Radio observational constraints on Galactic 3D-emission models
}

\author{
X. H. Sun ${ }^{1, \star}$, W. Reich ${ }^{1}$, A. Waelkens ${ }^{2}$, and T. A. Enßlin ${ }^{2}$ \\ 1 Max-Planck-Institut für Radioastronomie, Auf dem Hügel 69, 53121 Bonn, Germany \\ e-mail: wreich@mpifr-bonn.mpg.de \\ ${ }^{2}$ Max-Planck-Institut für Astrophysik, Karl-Schwarzschild-Str. 1, 85741 Garching, Germany
}

Received 14 September 2007 / Accepted 7 November 2007

\begin{abstract}
Context. Our position inside the Galaxy requires 3D-modelling to obtain the distribution of the Galactic magnetic field, cosmic-ray (CR) electrons and thermal electrons.

Aims. Our intention is to find a Galactic 3D-model which agrees best with available radio observations.

Methods. We constrain simulated all-sky maps in total intensity, linear polarization, and rotation measure (RM) by observations. For the simulated maps as a function of frequency we integrate in $15^{\prime}$ wide cones the emission along the line of sight calculated from Galactic 3D-models. We test a number of large-scale magnetic field configurations and take the properties of the warm interstellar medium into account.

Results. From a comparison of simulated and observed maps we are able to constrain the regular large-scale Galactic magnetic field in the disk and the halo of the Galaxy. The local regular field is $2 \mu \mathrm{G}$ and the average random field is about $3 \mu \mathrm{G}$. The known local excess of synchrotron emission originating either from enhanced CR electrons or random magnetic fields is able to explain the observed high-latitude synchrotron emission. The thermal electron model (NE2001) in conjunction with a proper filling factor accounts for the observed optically thin thermal emission and low frequency absorption by optically thick emission. A coupling factor between thermal electrons and the random magnetic field component is proposed, which in addition to the small filling factor of thermal electrons increases small-scale RM fluctuations and thus accounts for the observed depolarization at $1.4 \mathrm{GHz}$.

Conclusions. We conclude that an axisymmetric magnetic disk field configuration with reversals inside the solar circle fits available observations best. Out of the plane a strong toroidal magnetic field with different signs above and below the plane is needed to account for the observed high-latitude RMs. The large field strength is a consequence of the small thermal electron scale height of $1 \mathrm{kpc}$, which also limits the CR electron extent up to a height of $1 \mathrm{kpc}$ not to contradict with the observed synchrotron emission out of the plane. Our preferred 3D-model fits the observed Galactic total intensity and polarized emission better than other models over a wide frequency range and also agrees with the observed RM from extragalactic sources.
\end{abstract}

Key words. polarization - radiation mechanisms: non-thermal - radiation mechanisms: thermal - ISM: magnetic fields -

ISM: structure - Galaxy: structure

\section{Introduction}

Synchrotron radiation is the major component of the Galactic radio continuum emission from centimetre to longer wavelengths. The diffuse Galactic synchrotron emission originates from relativistic electrons gyrating in the magnetic field. An additional smaller contribution is observed from supernova remnants (SNRs) and pulsar wind nebulae located in the Galactic plane, where cosmic ray (CR) electrons are accelerated to high energies. Synchrotron emission is intrinsically highly polarized, but Faraday rotation effects in the magnetized interstellar medium along the line of sight and also beam averaging effects reduce the observed percentage polarization to much smaller values. It is crucial to understand the regularity, strength and spatial structure of the magnetic field in our Galaxy, which are still under discussion although the first detection of Galactic polarization was several decades ago (Wielebinski et al. 1962; Westerhout et al. 1962). Our position inside the Galactic disk at about $8.5 \mathrm{kpc}$ distance from the Galactic centre requires modelling of the magnetic field and CR electrons to interpret observed synchrotron

* Current address: National Astronomical Observatories, Chinese Academy of Sciences, Jia-20 Datun Road, Chaoyang District, Beijing 100012, PR China, e-mail: xhsun@bao.ac.cn. maps. Polarization maps are influenced by Faraday rotation effects along the line of sight and thus require to take the distribution of thermal electrons in the Galaxy into account as well. Important information on the Galactic magnetic field strength and direction comes from new rotation measure (RM) data. All these information must be commonly considered to constrain Galactic 3D-models in order to find the most realistic one. This paper presents a general attempt in that direction.

Previous 3D-models for the magnetic field, CR electrons and thermal electrons were derived individually from selected data sets. Their general relevance can be established by combining these models to explain other, in particular new observational data, for instance from WMAP. Here we consider RMs from extragalactic sources (EGSs), thermal and non-thermal intensities and linearly polarized emission together, which proves to give stringent constraints to combined 3D-models. We note that simulated maps are also required as a robust reference for the technical design of future telescope projects like the SKA or as inputs for data reduction pipelines like that for the PLANCK mission.

The paper is organized as follows: we review the status of Galactic models in Sect. 2, describe the method of our simulations in Sect. 3, summarize available observations used to constrain the 3D-models in Sect. 4 and briefly describe numerical 
details of the simulations in Sect. 5. The 3D-model results are presented in Sect. 6. Some general problems relevant to the modelling are discussed in Sect. 7. Conclusions are presented in Sect. 8.

\section{Status of galactic 3D-models}

Many attempts have been made to recover the structure of the Galactic synchrotron emission. Beuermann et al. (1985) deconvolved the $408 \mathrm{MHz}$ all-sky total intensity map (Haslam et al. 1982) and modelled the emission as a thick disk with a kpc scale height contributing about $90 \%$ of the total emission plus an embedded thin disk with a scale height of about 200 pc, which is consistent with the results by Phillipps et al. (1981a,b) using the same data. They adapt a spiral pattern for the emissivity and assume comparable regular and random magnetic field strength. Fleishman \& Tokarev (1995) present observational evidence for an excess of synchrotron emissivity within a few hundred parsec of the solar system based on low-frequency absorption data towards a number of large HII regions. A recent study of local Faraday screens by Wolleben \& Reich (2004) seems to confirm this result. Since local emission is also visible at high Galactic latitudes the thick disk assumption might be re-discussed.

The observed spectrum of Galactic synchrotron emission shows complex spatial variations due to processes such as energy losses, diffusion of CR electrons or variations of the magnetic field strength, which makes a modelling of the spectral behaviour rather difficult. For the northern sky Reich \& Reich (1988a,b) found spectral variations $\left(T_{v} \propto v^{\beta}\right.$, with $T_{v}$ the brightness temperature at frequency $v$ and $\beta$ the temperature spectral index) in the range $\beta \sim-3.1$ to $\beta \sim-2.5$ between $408 \mathrm{MHz}$ and $1420 \mathrm{MHz}$. At lower frequencies the spectrum is flatter. Roger et al. (1999) find spectral variations between $\beta \sim-2.55$ and $\beta \sim-2.4$ between $22 \mathrm{MHz}$ and $408 \mathrm{MHz}$. At higher frequencies the synchrotron spectrum steepens, but the amount is unclear so far. For the $1420 \mathrm{MHz}$ and $22.8 \mathrm{GHz}$ surveys Reich et al. (2004) calculate steeper spectra towards higher latitudes than below $1420 \mathrm{MHz}$. However, the $22.8 \mathrm{GHz}$ (K-band) WMAP data (Hinshaw et al. 2007) may contain an unknown fraction of anomalous dust emission (Davies et al. 2006) and thus the synchrotron spectrum might be steeper than the total emission spectrum. For any modelling it implies that the assumption of a constant spectral index is a simplification.

Synchrotron emission is generated by CR electrons in the Galactic magnetic field, which are considered as independent. We are unfortunately located in the disk of our Galaxy, which makes the observation of the large-scale field difficult. From observations of other spiral galaxies it is known that the field basically follows a spiral pattern (Beck et al. 1996). There are many methods to obtain information about the Galactic magnetic field observationally (see e.g. Han \& Wielebinski 2002). A powerful method is the analysis of pulsar RMs, which indicates from recent data that the field direction is opposite in the arm and the inter-arm regions (Han et al. 2006). However, RM data from EGSs can be interpreted with less reversals (Brown et al. 2003b; Haverkorn et al. 2006; Brown et al. 2007). The local regular field strength is about $2 \mu \mathrm{G}$ and increases to about $4 \mu \mathrm{G}$ at about $3 \mathrm{kpc}$ from the Galactic centre according to Han et al. (2006), where no coupling between the magnetic field strength and the thermal electron density is assumed. Strong et al. (2000) estimated the total local field to be about $6 \mu \mathrm{G}$. The Galactic random field component was estimated to be about $6 \mu \mathrm{G}$ (Han et al. 2006). This is about a factor of 2 to 3 larger than the regular field component. This ratio is larger than it was assumed in earlier models.
The distribution of Galactic CR electrons has still to be established. The emission at radio frequencies originates from $\mathrm{GeV}$ electrons, where a power law distribution is a good approximation. SNRs are the acceleration sites and thus the sources of CR electrons. However, the propagation of CRs in our Galaxy is very complex and modelling efforts like GALPROP (Strong \& Moskalenko 1998) are required.

The properties for the magnetized thermal interstellar medium are crucial for the interpretation of polarization observations, because Faraday effects strongly modulate the polarization emission along the line of sight and make modelling of Galactic polarization quite challenging. At low frequencies Faraday effects might be strong enough to entirely smear out distant polarization (Sokoloff et al. 1998). For the Galactic large-scale thermal component the NE2001 model based on pulsar dispersion measures (Cordes \& Lazio 2002) represents the best knowledge. However, additional Faraday effects come from HII regions and also from Faraday screens hosting strong regular fields, but a low thermal electron density (Wolleben \& Reich 2004; Sun et al. 2007; Reich 2006), and might influence the observations by a yet unknown amount.

\section{The method applied}

The observables are RM data, total and polarized intensity maps at different frequencies. Polarization angles and percentage polarization can be used also for constraints. The goal is to obtain the thermal electron, CR electron and magnetic field 3D-distribution in the Galaxy which agrees best with the observations.

RMs depend on the regular magnetic field component and the thermal electron density along the line of sight. The perpendicular magnetic field component and the CR electron density determine the total intensity synchrotron emission. Observed polarized intensity depends on the regular fraction of the magnetic field, but gets modified as a function of frequency by the line of sight magnetic field components and the thermal electron density. To achieve a "best model" an optimized quantity like $\chi^{2}$ should be used to adjust the model parameter. This is beyond the scope of the present attempt and also clearly needs more observations than actually available for a well established model.

We tackle the 3D-modelling problem by finding the model parameters in four steps as shown in Fig. 1. The modelling is based on the thermal electron density distribution as it is described by the widely accepted NE2001 model (Cordes \& Lazio 2002). After considering a proper filling factor for the thermal electron distribution of the NE2001 model we are able to reproduce the observed optically thin free-free emission and the effect of absorption from the optically thick gas. This indicates that the NE2001 model is valid in general, although a larger scale height is favoured by our modelling results as discussed later. From RM data of EGSs we are able to obtain the regular magnetic field direction and its strength separately for the Galactic plane and towards higher latitudes by keeping the thermal electron model parameters from NE2001 fixed. From the observed $408 \mathrm{MHz}$ total intensity and the $22.8 \mathrm{GHz}$ polarized intensity we constrain the random magnetic field component and the large-scale distribution of CR electrons using the regular magnetic field component obtained in the step before. If the random field component is increased or decreased the CR electron density must be reduced or enlarged accordingly to fit the total intensity emission. Both components must be matched to fit the observed polarized intensity. 


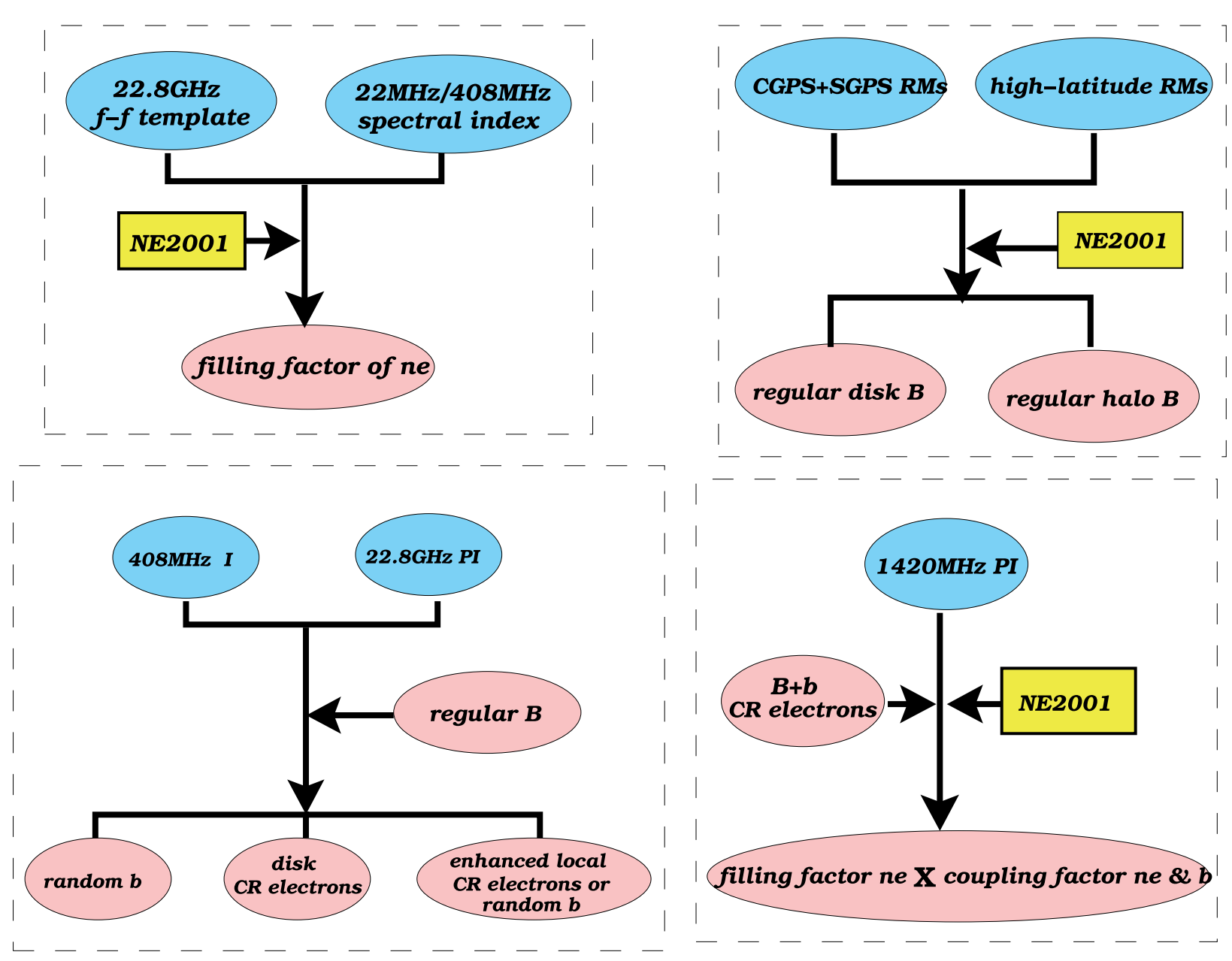

Fig. 1. Overview on the different steps applied to find the Galactic 3D-emission components and their properties. Here f-f stands for free-free emission, ne for thermal electons, $\boldsymbol{B}(\boldsymbol{b})$ vectors for the regular (random) magnetic field component, and I (PI) for total (polarized) intensity. CGPS and SGPS mean the Canadian and the Southern Galactic Plane Survey, respectively. The blue color is for observational data, the pink for the model and the yellow for NE2001.

The local excess of synchrotron emission is considered in our model, which is able to account for the observed highlatitude emission as well. At that point our model is able to represent the total intensity synchrotron emission and the intrinsic polarization properties as observed at high frequencies and is in general agreement with the observed extragalactic RM distribution. In the last step, the observed depolarization properties at $1.4 \mathrm{GHz}$ are accounted for by introducing a coupling factor between the thermal electron density and the magnetic field strength on small-scales in addition to the thermal filling factor.

\section{Available observations constraining the 3D-models}

To constrain the simulated maps from Galactic 3D-models we consider various observed all-sky data sets. These maps contain the integrated line of sight information of large-scale Galactic emission, but also show all kinds of local foreground structures and distinct source complexes, which are not the subject of modelling in our case. We note that it is not always clear how to distinguish and to correctly separate the observed structures into local and global emission components. Unresolved strong sources can be easily identified and are not represented by the models. Large-scale local features like the giant radio loops with diameters up to $100^{\circ}$ clearly show up as distinct feature in the total intensity and the polarization maps and are also not included in the model, although they are difficult to separate from the observed maps. The large-scale highly polarized "Fan region" is difficult to trace in total intensities, but certainly contributes to the observed emission. Distinct SNRs and large HII region complexes are concentrated in the Galactic plane and are excluded from the modelling, but their position is known from highresolution surveys along the Galactic plane. Faraday screens of considerable size exist in the interstellar medium, which are weak emitters, but reflect their existence by their large RMs by rotating the polarization angles of the background emission. Only a very densely sampled RM grid of sources or multifrequency observations of diffuse Galactic polarization can reveal such features. These data are not available, but their importance is widely accepted. "Key science projects" for LOFAR and the future SKA were defined (Gaensler et al. 2004), which exactly have these aims. Faraday screens might influence our modelling results to an unknown extent. All the uncertainties in the recognition of local or distinct features limits any Galactic modelling attempt to the reconstruction of the large-scale Galactic structures.

\subsection{All-sky total intensity maps}

All-sky total intensity surveys up to a few $\mathrm{GHz}$, which are adjusted to an absolute zero-level, are essential to obtain the 
required large-scale knowledge of the Galactic synchrotron emission. Carrying out such surveys is rather time-consuming and therefore these surveys have relatively low angular resolution. Available all-sky surveys have been reviewed by Reich (2003). Among these the $408 \mathrm{MHz}$ survey (Haslam et al. 1982) and the $1420 \mathrm{MHz}$ survey (Reich 1982; Reich \& Reich 1986; Reich et al. 2001) have the highest sensitivity and an angular resolution down to 0.6 and thus are well suited for our purpose.

Galactic radio emission at frequencies below a few $\mathrm{GHz}$ is dominated by synchrotron emission, in particularly out of the plane, relative to free-free emission. Thermal dust emission and probably spinning dust emission are becoming important for frequencies higher than $5 \mathrm{GHz}$ or $10 \mathrm{GHz}$. The $408 \mathrm{MHz}$ total intensity map is often used as a "synchrotron template" and in fact significant thermal emission is confined to the Galactic plane. At $1420 \mathrm{MHz}$ the thermal fraction in the plane increases to about $40 \%$ in the inner Galaxy (Reich \& Reich 1988b). Paladini et al. (2005) estimated a thermal contribution with a maximum of about $68 \%$ for the region of $20^{\circ}<l<30^{\circ}$ and $|b|<1.5$, where discrete HII regions contribute about $9 \%$. Note that these estimates are based on the separation of thermal and non-thermal components assuming appropriate spectral indices. However, the spectral index of synchrotron emission is uncertain and might vary with direction, although these estimates are largely consistent with the method to separate both components based on a relation between infrared and thermal emission (Broadbent et al. 1989). It is certainly worth to refine the separation of thermal and non-thermal emission, but this is beyond the scope of the present paper.

Dickinson et al. (2003) have constructed an extinction corrected free-free emission template for about $95 \%$ of the sky based on $\mathrm{H} \alpha$ surveys of the northern sky (Haffner et al. 2003) and of the southern sky (Gaustad et al. 2001) by using dust emission data (Schlegel et al. 1998) to calculate the extinction correction. Hinshaw et al. (2007) used the $\mathrm{H} \alpha$ template by Finkbeiner (2003) as a prior estimate in the maximum entropy method analysis of the 3-year WMAP data to construct the free-free emission template. This template might contain some leakage from other components such as synchrotron emission and anomalous dust emission. However, actually it probably represents the best estimate for low latitude emission. We will refer to the freefree emission map at $22.8 \mathrm{GHz}$ from Hinshaw et al. (2007) for comparison with the modelled thermal emission. The Dickinson et al. (2003) template cannot be used for the Galactic plane area because of the large and uncertain extinction there.

All radio maps show a clear concentration of emission along the Galactic plane. As mentioned above, local structures out of the plane are rather dominant features. The most striking protrusion emerging at $l \sim 30^{\circ}$ out of the plane is called the North Polar Spur (NPS), which can be traced up to very high latitudes. This spur is a part of the giant Loop I, which is believed to be a local old supernova remnant at a distance of about $200 \mathrm{pc}$ (Berkhuijsen 1973). A recent new model by Wolleben (2007) tries to explain the NPS in total intensity and linear polarization by local synchrotron emitting shells with a swept-up magnetic field. A number of weaker loops of similar size exist as discussed by Berkhuijsen et al. (1971).

\subsection{All-sky polarization maps}

The status of Galactic polarization surveys has been recently reviewed by Reich (2006). For the interpretation of polarization surveys the correct absolute zero-level is of particular importance as it was demonstrated by Reich et al. (2004). The only
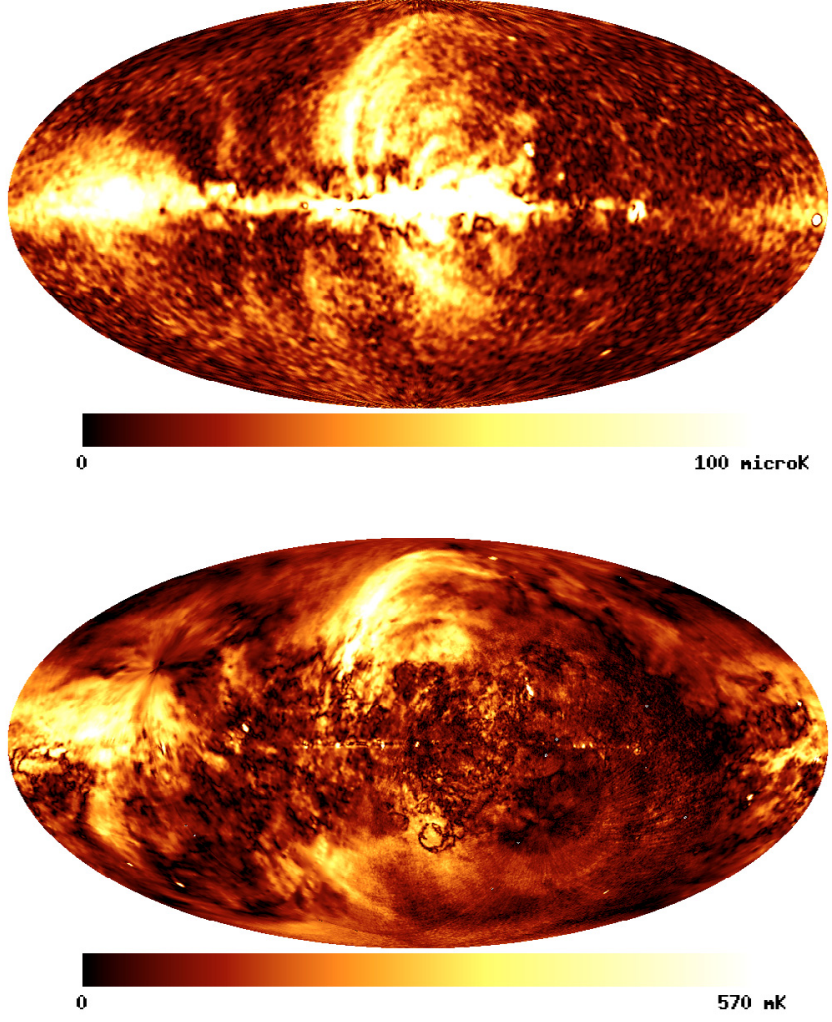

Fig. 2. The WMAP $22.8 \mathrm{GHz}$ all-sky polarized intensity map (upper panel) and the $1.4 \mathrm{GHz}$ all-sky polarized intensity map (lower panel). The polarized intensities are shown greyscale coded from 0 to $100 \mu \mathrm{K}$ for $22.8 \mathrm{GHz}$ and from 0 to $570 \mathrm{mK}$ for $1.4 \mathrm{GHz}$.

ground based all-sky polarization map exists at $1.4 \mathrm{GHz}$ (Reich et al., in prep.) combining the northern sky survey carried out with the DRAO 26-m telescope (Wolleben et al. 2006), which is tied to the absolute level of the $1.4 \mathrm{GHz}$ Leiden survey (Brouw \& Spoelstra 1976), and the Villa Elisa southern sky survey (Testori et al. 2004; Testori et al., in prep.), which has a large overlap with the DRAO survey. At this frequency the depolarization effects are already very strong at latitudes below about $30^{\circ}$, especially towards the Galactic plane as can be seen from Fig. 2. Thus the distance up to which polarized emission can be observed at $1.4 \mathrm{GHz}$ with an angular resolution of $36^{\prime}$ is small compared to the line of sight through the Galaxy. On the other hand the WMAP $22.8 \mathrm{GHz}$ polarized intensity map (Page et al. 2007) is almost unaffected by Faraday depolarization effects and therefore should represent the intrinsic synchrotron properties of the Galaxy, since dust polarization is believed to be entirely unimportant at that frequency (Page et al. 2007).

As it can be seen from the $22.8 \mathrm{GHz}$ map there are strong imprints of Loop I, which shows strong polarization along the NPS and also below the Galactic plane. Loop I is very local as mentioned in the previous section and therefore can be also traced for large sections at $1.4 \mathrm{GHz}$. Another very bright polarization feature called the "Fan region" is centered at Galactic longitude of about $140^{\circ}$ and was recognized as a local feature at a distance of about 500 pc (Wilkinson \& Smith 1974; Brouw \& Spoelstra 1976). However, a new analysis based on the DRAO $1.4 \mathrm{GHz}$ survey suggests that it might extend from about $500 \mathrm{pc}$ to about $2 \mathrm{kpc}$ (Wolleben et al. 2006). We take both structures as distinct local features, as they are very bright at $1.4 \mathrm{GHz}$ contrary to the polarized emission from the Galactic plane. The modelling 
of these distinct features is beyond the scope of our simulation. They will not influence our modelling of the large-scale Galactic structures.

Although external Faraday depolarization is very small at $22.8 \mathrm{GHz}$ we find a low percentage polarization at $22.8 \mathrm{GHz}$ in general. This is in agreement with the results of Kogut et al. (2007). We divided the polarized intensity map by the synchrotron emission template provided by the WMAP team. The polarization percentage in the Galactic plane is typically below $2 \%$. At higher latitudes typical values between $12 \%$ and $30 \%$ are derived, which are rather similar to those found at $1.4 \mathrm{GHz}$. Of course, the $22.8 \mathrm{GHz}$ percentage polarizations may be systematically underestimated in case of the existence of a significant spinning dust component in the total intensity map. Despite of this uncertainty the intrinsic synchrotron polarization will stay significantly below the maximum polarization value for a perfect regular field. This might indicate that the random magnetic field exceeds the regular magnetic field component. Otherwise the superposition of a number of regular magnetic field components along the line of sight with different orientations may have the same effect.

Comparing the $22.8 \mathrm{GHz}$ and the $1.4 \mathrm{GHz}$ polarized emission we see strong depolarization effects along the Galactic plane except for the "Fan region". This depolarization is attributed to Faraday effects from the warm ionized medium. However, it is not immediately clear, whether the diffuse medium or sources like HII regions or Faraday screens play the main role in this process. We will show below that the depolarization calculated from the diffuse thermal component as provided by the NE2001 model is insufficient to explain the $1.4 \mathrm{GHz}$ polarization observations.

The polarized intensity can be used to constrain the strength of the regular magnetic field. As can be seen from the $22.8 \mathrm{GHz}$ polarization map (Fig. 2) the main characteristics are (1) strong polarization towards the Galactic centre; (2) the distribution of polarized emission along Galactic longitude is asymmetric with respect to the Galactic centre. Depolarization can be neglected at $22.8 \mathrm{GHz}$ and therefore the polarization angles could also be used to check the orientation of the magnetic field. However, our simulations show that any spiral magnetic field configuration gives rather similar polarization angle patterns. Therefore we do not use polarization angles to decide on the field configuration as it was previously done by Page et al. (2007). As shown below this model is inconsistent with other observations.

\subsection{Rotation measures of extragalactic sources}

When a linearly polarized electromagnetic wave passes a magnetized thermal medium its polarization angle experiences rotation, called Faraday rotation. The rotation is proportional to the square of the wavelength and the coefficient is the RM, which can be written as

$\mathrm{RM}=0.81 \int_{0}^{D} n_{\mathrm{e}}(l) B_{\|}(l) \mathrm{d} l$

where the integral range is along the line of sight from the observer to the source at a distance $D$ measured in pc. $n_{\mathrm{e}}$ per $\mathrm{cm}^{-3}$ is the thermal electron density and $B_{\|}$in $\mu G$ is the magnetic field component along the line of sight in the intervening medium. RM is positive when the magnetic field points towards us.

RMs of EGSs reflect the integrated effect of the magnetized medium throughout the Galaxy and can be used for a diagnosis of the thermal electron density and magnetic field strength along

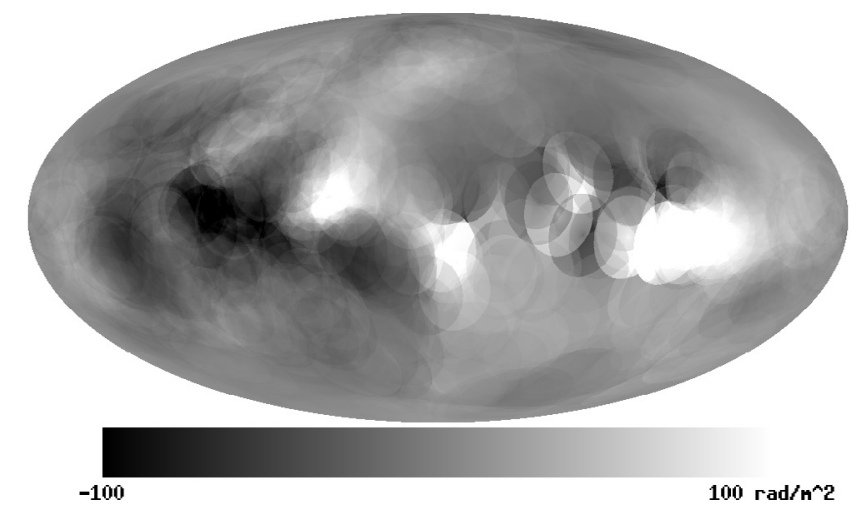

Fig. 3. Synthesized RM map constructed from published and new RM data of EGSs (Han et al., in prep.). The preliminary new RM data set from the Effelsberg survey was provided by JinLin Han. The RM map does not include Galactic plane RM data from the Canadian (CGPS) or the Southern Galactic Plane (SGPS) surveys.

the line of sight. The RM contribution from outside the Galaxy should be very small. The effect of the EGS-intrinsic RM can only be accounted for by averaging a sufficient large number of RMs in a small area. Unfortunately RM data of EGSs are sparsely distributed in the sky despite recent observational efforts. This holds in particular for the southern sky, which makes the construction of an all-sky RM template a difficult task. With the RM data compiled by Han et al. (1999) and the preliminary RM data from the recent $L$-band Effelsberg RM survey of about 1800 polarized NVSS sources (Han et al., in prep.) we synthesized the RM all-sky map shown in Fig. 3, following the same interpolation method as used by Uyanıker et al. (1998) to create a smooth map from ungridded data. The observed data close to each pixel of the interpolated all-sky RM map are added with a weight calculated from the function $\exp \left(-(\theta / \Theta)^{m}\right)$, where $\theta$ is the angular distance to the pixel. $\Theta$ was set to $8^{\circ}$ and $m$ to 0.3 . This RM map contains more data than used by Dineen $\&$ Coles (2005) and Johnston-Hollitt et al. (2004). However, the basic large-scale features seen in all RM maps are rather similar. Although there are less data in the southern sky, which causes edge effects as visible in Fig. 3, the data clearly indicate the general RM trend as discussed below. We did not include the numerous RM data by Brown et al. (2003a, 2007) from sources in the Galactic plane as the intention is to trace the high-latitude RM characteristics. We treat the Galactic plane RM characteristics separately.

The RM map in general shows an antisymmetric distribution below and above the Galactic plane and also with respect to Galactic longitude $0^{\circ}$, as already noted by Han et al. (1997). In a recent RM study towards the Galactic centre region this RM pattern was also seen towards the inner Galaxy (Law 2007). The high-latitude RM values are about $20-30 \mathrm{rad} \mathrm{m}^{-2}$ on average.

For the RM distribution along the Galactic plane we rely on the EGSs detected in high-resolution Galactic plane surveys, where sources contrast well against the Galactic diffuse emission. The Canadian Galactic plane survey (CGPS, Taylor et al. 2003) covers parts of the northern sky. The southern Galactic plane is covered by the SGPS (Gaensler et al. 2001). The RM data from the CGPS (Brown et al. 2003a) and the SGPS (Brown et al. 2007) are shown in Fig. 4. Note that the fluctuation of RMs is very large, typically around several hundred $\mathrm{rad} \mathrm{m}^{-2}$, and averaging over large areas is needed to trace systematic $\mathrm{RM}$ changes. We sort the data into longitude bins and obtain 


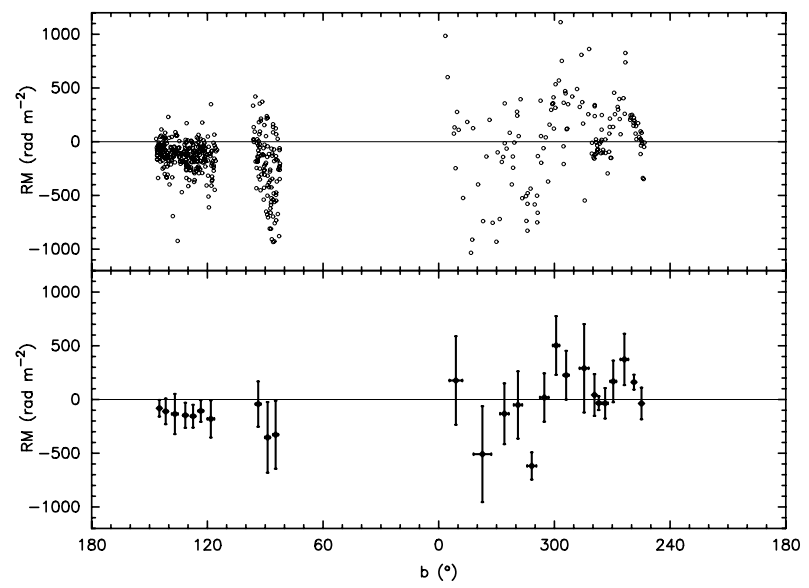

Fig. 4. RM profiles along the Galactic plane with RM data for the northern Galactic plane taken from Brown et al. (2003a) and RM data for the southern Galactic plane from Brown et al. (2007). The average for each longitude bin is shown in the lower panel. The bars indicate the $\pm 1 \sigma$ variance.

their average to be compared with the simulations. For the SGPS data the binning procedure is the same as used by Brown et al. (2007). For the CGPS data, the RMs between $l=82^{\circ}$ and $l=96^{\circ}$ are divided into three bins containing 38 or 39 sources and the RMs elsewhere are put into 11 bins containing 37 or 38 sources. The binned data are also shown in Fig. 4. We note the following RM structural features: (1) the average RMs run smoothly from about $-100 \mathrm{rad} \mathrm{m}^{-2}$ at $l \approx 140^{\circ}$ to about $-200 \mathrm{rad} \mathrm{m}^{-2}$ at $l \approx 100^{\circ} ;(2)$ the sign of the RMs changes at $l \approx 304^{\circ}$. We believe these features to be real. The local minimum of RMs at $l \approx 310^{\circ}$ seems also reliable. There are some more features, which seem less certain like $\mathrm{RM}$ values close to zero appearing at $l \approx 320^{\circ}$ and $l \approx 275^{\circ}$. The RM fluctuations in the Galactic centre region are extremely large (Roy et al. 2005), which seem to reflect strong variations of the properties of the interstellar medium located in the centre region. The available amount of RM data is insufficient to allow to constrain the large-scale properties of the interstellar medium and the magnetic field in the central region of the Galaxy.

The large RM fluctuations observed might originate from the intrinsic RMs of the EGSs or the interstellar medium in the Galaxy. Fluctuations of the Galactic magnetic field have been estimated to be about $6 \mu \mathrm{G}$ (Han et al. 2004), which might cause the large scatter of RMs. However, we will show below that the clumpiness of thermal electrons and a likely correlation with the random magnetic field component causes large enough RM fluctuations for depolarization in accordance with the observations. We note that strong RM fluctuations are a handicap for the detection of cosmological magnetic fields from fluctuations of foreground corrected RMs as reviewed by Widrow (2002).

Currently the RMs of pulsars are not considered for modelling. First of all the distances of pulsars are quite uncertain as many of them are obtained from the dispersion measure based on global electron density models, e.g. NE2001. Second, our simulation results are all-sky maps, which are difficult to compare with the RMs of pulsars, which are distributed along the line of sight. Finally, despite of recent efforts, pulsar RM data are still sparsely distributed in the Galactic plane and it is difficult to account for the influence of the local interstellar medium. In future the pulsar RM data definitely will be used as important additional constraints in particular as they have no intrinsic RM contribution.

\section{Numerical realization of Galactic 3D-modelling}

Waelkens (2005) developed the HAMMURABI code aiming to simulate the all-sky synchrotron emission of the Galaxy. The code takes Faraday rotation effects into account and thus Faraday depolarization effects are naturally involved. In this work we implemented additional entries to account for optically thick freefree emission. The code output maps follow the HEALPIX pixelization scheme (Górski et al. 2005), which divides the sky into pixels of equal areas. The total number of pixels for an all-sky map, which defines its angular resolution, is $N_{\text {pix }}=12 N_{\text {SIDE }}^{2}$, with $N_{\mathrm{SIDE}}=2^{k}$ and $k=0,1,2,3, \ldots$ The angular size of a pixel $(\Delta \theta)$ can be approximated as,

$\Delta \theta \approx \sqrt{\frac{3}{\pi}} \frac{3600^{\prime}}{N_{\mathrm{SIDE}}}$.

In our simulation we use $N_{\mathrm{SIDE}}=256$, which corresponds to an angular resolution of $15^{\prime}$. This selection is a compromise between angular resolution and computing time.

Along the line of sight the cone for each pixel is divided into volume elements in radial direction, where in this work each unit has an equal radial length $\Delta r=20 \mathrm{pc}$. The largest volume unit in such a cone is approximately

$V_{\max }=4 \pi r_{\max }^{2} \frac{\Delta r}{12 \cdot N_{\text {side }}^{2}}$,

where $r_{\max }$ is the longest possible line of sight with the maximal value of $32 \mathrm{kpc}$. Due to the disc like shape of our galaxy the effective distance out to which we perform our computations varies and decreases sharply towards high latitudes and towards the anti-centre direction. Note that fluctuations of the magnetic fields on scales smaller than the volume unit cannot be resolved by the code.

For the $i$ th volume element of a cone we compute the following quantities,

$$
\left\{\begin{aligned}
I_{i}^{\mathrm{syn}} & =C_{I} B_{i, \perp}^{(1-p) / 2} v^{(1+p) / 2} \Delta r \\
P I_{i} & =C_{P I} B_{i, \perp}^{(1-p) / 2} v^{(1+p) / 2} \Delta r \\
R M_{i} & =0.81 n_{\mathrm{e} i} B_{i, \|} \Delta r \\
\psi_{i} & =\psi_{i, 0}+\sum_{1}^{i} R M_{j} \lambda^{2} \\
U_{i} & =P I_{i} \sin \left(2 \psi_{i}\right) \\
Q_{i} & =P I_{i} \cos \left(2 \psi_{i}\right) \\
E M_{i} & =n_{\mathrm{e} i}^{2} \Delta r \\
\tau_{i} & =8.235 \times 10^{-2} T_{i}^{-1.35} v^{-2.1} E M_{i} \\
I_{i}^{\mathrm{ff}} & =T_{i}\left(1-\exp \left(-\tau_{i}\right)\right) .
\end{aligned}\right.
$$

Note that the calculated Stokes values $U_{i}$ and $Q_{i}$ for each volume element include the effect of foreground RM. The CR electrons are assumed to follow a power law with an energy spectral index $p$. The values $C_{I}$ and $C_{P I}$ depend on the spectral index $p$ and are further detailed in Rybicki \& Lightman (1979). The synchrotron emission is related to the magnetic field component perpendicular to the line of sight $B_{i, \perp}$, while the RM depends on the magnetic field parallel to the line of sight $B_{i, \|}$. The intrinsic polarization angle in each volume element is $\psi_{i, 0}$, which is defined as the inclination angle of $B_{i, \perp}$ with respect to the north (in the frame of Galactic coordinates). Note that the magnetic field is the sum of the regular and random field components. The thermal electron density is $n_{\mathrm{e} i}$ and its temperature is $T_{i}$. For the 


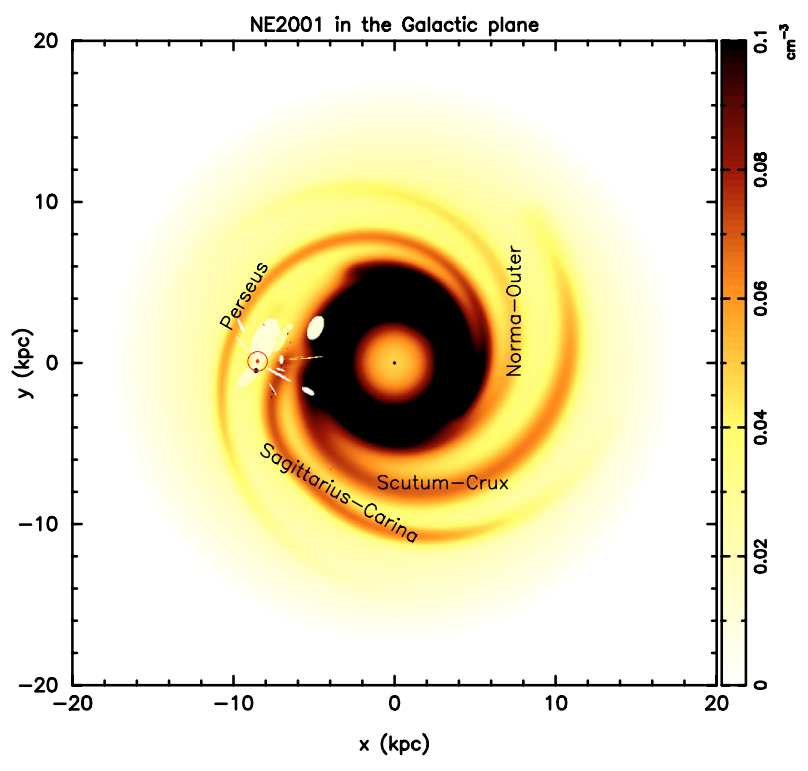

Fig. 5. The NE2001 thermal electron density model by Cordes \& Lazio $(2002,2003)$ projected on the Galactic plane. The position of the Sun as well as the designation of the spiral arms are indicated.

relation between opacity $\tau_{i}$ and emission measure, EM, we refer to Rohlfs \& Wilson (2000). The calculation of $E M_{i}, \tau_{i}$ and $I_{i}^{\mathrm{ff}}$ are expansions of the original HAMMURABI code.

Intensities and RMs for each pixel are obtained as the integral of the contributions from all the volume units along the line of sight

$$
\left\{\begin{aligned}
I^{\mathrm{syn}} & =\sum_{i} I_{i}^{\mathrm{syn}} \\
I^{\mathrm{ff}} & =\sum_{i} I_{i}^{\mathrm{ff}} \\
Q & =\sum_{i} Q_{i} \\
U & =\sum_{i} U_{i} \\
R M & =\sum_{i} R M_{i} .
\end{aligned}\right.
$$

All intensities are calculated as a function of frequency. More details on the HAMMURABI code will be discussed in a forthcoming paper by Waelkens et al. (in prep.).

\section{Modelling}

For the description of the 3D-models we introduce cylindrical coordinates $(R, \phi, z)$, where $R$ is the Galactocentric radius, $\phi$ is the azimuth angle starting from $l=180^{\circ}$ and increasing in anticlockwise direction, and $z$ is the distance to the Galactic plane. To show the slices of the 3D models a Galactocentric Cartesian coordinate is also used, where $z$ is the same as in the cylindrical system, and the $x-y$ plane coincides to the Galactic plane with $x$ pointing to $l=0^{\circ}$ and $y$ to $l=90^{\circ}$. Through all calculations the distance of the Sun to the Galactic centre is taken as $8.5 \mathrm{kpc}$.

Below we present a detailed 3D modelling of the Galactic emission following the steps described in Sect. 3.

\subsection{Thermal electron density}

\subsubsection{The model for diffuse ionized gas - NE2001}

According to Ferrière (2001) the warm ionized medium (WIM) has an electron density of $0.2 \mathrm{~cm}^{-3}$ to $0.5 \mathrm{~cm}^{-3}$, a volume filling factor of $5 \%$ to $14 \%$ and a temperature of about $8000 \mathrm{~K}$. It consists of HII regions embedded in diffuse ionized gas (DIG). The average electron density of HII regions can be derived from its radio continuum integrated flux density (e.g. Schraml \& Mezger 1969). However, the distances of HII regions are required, which are for instance derived from observations of Doppler shifted recombination lines. Kinematic distances are based on Galactic rotation curves and are not quite certain. The scale height for HII regions is about $50 \mathrm{pc}$ in the inner Galaxy and increases towards larger radii (Paladini et al. 2004).

HII regions along the line of sight can influence RMs from sources in the background as shown by Mitra et al. (2003). Since HII regions are tightly confined to the Galactic plane, the chance that a line of sight passes an HII region outside the plane is small. In the Galactic plane the typical effect of HII regions on RMs is difficult to estimate in general. Both the electron density and the magnetic field inside a HII region may be an order of magnitude larger compared to the diffuse gas. A typical RM contribution of several tens of $\mathrm{rad} \mathrm{m}^{-2}$ was quoted by Mitra et al. (2003). These values are smaller compared to the average RM of EGs, which trace the entire magnetized DIG in the Galaxy along the line of sight. Therefore we consider only the effect of the DIG in our simulations.

A good tracer of the DIG is the dispersion measure (DM) of pulsars. The DM is defined as the integral of the electron density along the line of sight. Using pulsars with known distances as determined from e.g. parallax or HI-absorption measurements an electron density model can be obtained by a fit of the observed DMs. In this way a two-disk model was proposed and developed in detail by Cordes et al. (1991); Taylor \& Cordes (1993); Gómez et al. (2001). Cordes \& Lazio (2002) and Cordes $\&$ Lazio (2003) used more pulsars and improved the model by involving local irregularities and in particular added a spiral arm structure. Thus the NE2001 model has three components: a thick disk, a thin disk and spiral arms as displayed in Fig. 5. Locally the mid-plane electron density is about $0.03 \mathrm{~cm}^{-3}$ and the exponential scale height is about $0.97 \mathrm{kpc}$.

\subsubsection{The filling factor of the DIG - from free-free emission at $22.8 \mathrm{GHz}$}

The properties of the DIG can also be traced by its free-free emission. This is done either by measuring $\mathrm{H} \alpha$ intensities or radio continuum emission. However, the diffuse Galactic thermal and non-thermal radio emission components mix and are difficult to separate. $\mathrm{H} \alpha$ observations need an extinction correction to be a reliable free-free emission template (Dickinson et al. 2003), which is, however, only possible out of the plane. Because the distances of the emission components along the line of sight are unknown, the electron density distribution cannot be obtained directly. However, the free-free emission template can be used as a check to prove that the integrated properties of the DIG model are appropriate.

Before we calculate the all-sky free-free emission map from the NE2001 model we assess the contributions from HII regions. The thermal emission from HII regions is confined to a thin layer $\left(|b|<1^{\circ}\right.$ e.g. Paladini et al. 2004) along the Galactic plane with a small scale height. We do not include individual HII regions in our simulations of the free-free emission, which we believe is an acceptable simplification.

The electron temperature is required to calculate the freefree emission intensity as seen from Eq. (4). The existence of an electron temperature gradient in the Galaxy is known and was recently confirmed with some precision by Quireza et al. (2006) 

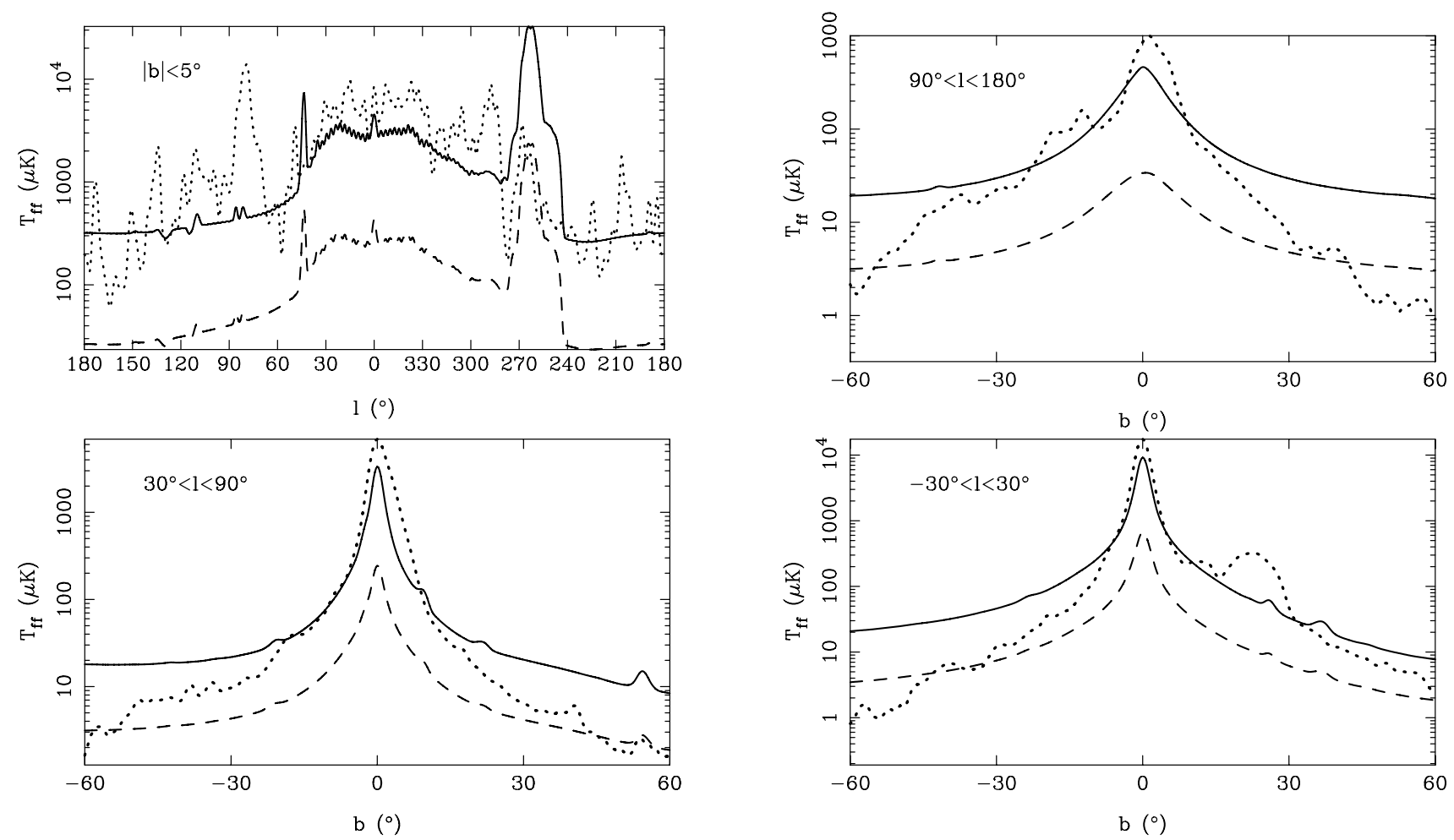

Fig. 6. Longitude and latitude profiles from the simulated and the free-free emission maps at $22.8 \mathrm{GHz}$. The dotted lines indicate the data from the 22.8 GHz template. The solid and dashed lines are from the thermal emission simulations based on the NE2001 model without (dashed) and with (solid) a filling factor correction according to Berkhuijsen et al. (2006).

from recombination line measurements as $T_{i}(R)=(5780 \pm 350)+$ $(287 \pm 46) R$, where $R$ is in units of kpc. This corresponds to an electron temperature of about $8000 \mathrm{~K}$ near the solar system, which is larger than that derived by Paladini et al. (2004). Note that these temperatures are obtained from observations of HII regions, which are known to be lower than the DIG by typically about 1000-2000 K (Madsen et al. 2006). However, these temperature differences have just a small influence on the amount of free-free emission (see Eq. (4)). For simplicity we use the temperature gradient established from the measurements of HII regions.

Reynolds et al. (1999) reported a temperature increase with increasing $z$. This increase was fitted by Peterson \& Webber (2002) as $T_{i}(z)=7000-526 z+1770 z^{2}$, where $z$ is in units of kpc. Combining both temperature gradients we obtain $T_{i}(R, z)=$ $(5780 \pm 350)+(287 \pm 46) R-526 z+1770 z^{2}$ being used in our calculation. Although the temperature is high at large $z$ the free-free emission remains small due to the low electron density there. Our results are not sensitive to the temperature gradient in $z$. We note that the electron gradient in $z$ direction is still debated. Recent results from Davies et al. (2006) based on WMAP and ancillary data indicate rather low electron temperatures at high latitudes.

When we calculate the free-free emission from the NE2001 model as shown in Fig. 5 by using Eq. (4), we obtain much smaller intensities than from the optically thin free-free emission template derived by Hinshaw et al. (2007) based on WMAP data. This can be seen from Fig. 6 where the longitude profiles (average for $|b|<5^{\circ}$ ) and the latitude profiles (average for the longitude ranges indicated in the plots) are shown. However, the differences vanish when the clumpy nature of the DIG is taken properly into account, which means to introduce an appropriate filling factor of the thermal electrons. Recently
Berkhuijsen et al. (2006) studied the Galactic filling factor $f_{\mathrm{e}}$ of the DIG and its dependence on $z$ in detail. The filling factor $f_{\mathrm{e}}$ is defined as $f_{\mathrm{e}}=\left\langle n_{\mathrm{e}}\right\rangle^{2} /\left\langle n_{\mathrm{e}}^{2}\right\rangle$, where the \langle\rangle means the average. Berkhuijsen et al. (2006) derived $f_{\mathrm{e}}(z)=0.07 \exp (|z| / 0.5)$, where $z$ is in units of $\mathrm{kpc}$, and $f_{\mathrm{e}}=0.32$ for $z$ larger than $0.75 \mathrm{kpc}$. EM calculated from the NE2001 model is based on $\left\langle n_{\mathrm{e}}\right\rangle$ and needs to be corrected by the factor $1 / f_{\mathrm{e}}$, which means that EM increases by a factor $1 / f_{\mathrm{e}}$. After employing the filling factor correction the comparison between the free-free emission template from WMAP observations and the corrected NE2001 model is shown in Figs. 6 and 7. The simulations now resemble the WMAP template quite well. From the comparison shown in Fig. 6 it is also obvious that the thermal high-latitude emission from the simulations is slightly higher than that from the template, which might indicate a further increase of the filling factor towards larger $z$ beyond the limit of 0.32 assumed by Berkhuijsen et al. (2006).

\subsubsection{The filling factor of the DIG - from low-frequency absorption}

At low frequencies the optical thickness of free-free emission increases (Eq. (4)) which implies that the synchrotron emission from behind is attenuated by a factor $\exp (-\tau)$. This can be seen by an apparent increase of the spectral index by $\tau / \ln \left(v_{h} / v_{l}\right)$, where $v_{h}$ and $v_{l}$ are the high and the low frequency, respectively. Since the optical thickness is proportional to EM the filling factor correction is essential. We simulate total intensity maps at $408 \mathrm{MHz}$ and $22 \mathrm{MHz}$ by using an observed spectral index of $\beta=-2.47$ obtained by Roger et al. (1999) from the $408 \mathrm{MHz}$ and $22 \mathrm{MHz}$ surveys for regions outside the Galactic plane, which closely represents the spectral index of the Galactic low frequency synchrotron emission. The spectral index map is calculated from the simulated maps at the two frequencies and the 

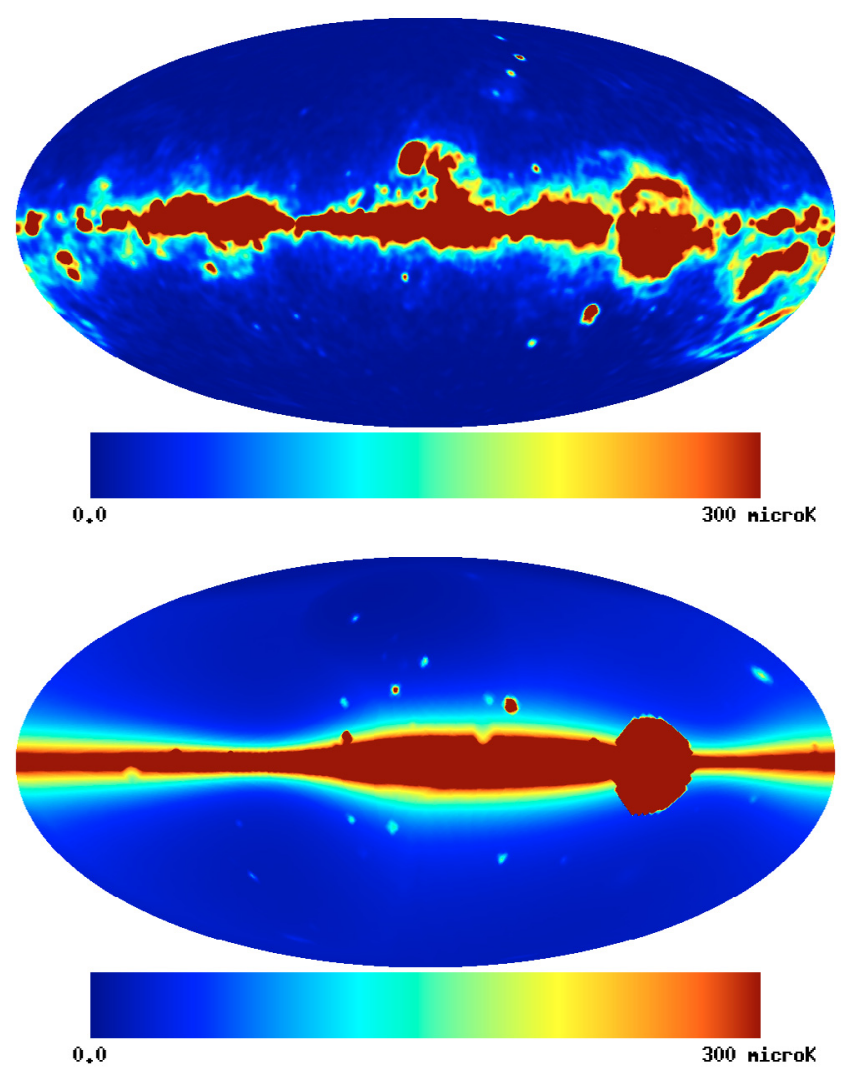

Fig. 7. The WMAP free-free emission template (Hinshaw et al. 2007) is shown in the upper panel and the NE2001 based simulated maps after taking a filling factor into account is shown in the lower panel. Both maps are shown at an angular resolution of $2^{\circ}$.

average profile along the Galactic plane is compared with that observed (Fig. 8). We find that the spectral index increase from $l=60^{\circ}$ towards the Galactic centre is well reproduced by our simulation. Note that the peaks are from individual source complexes, which are not included in our model. For comparison we show also the spectral index profile from the NE2001 model without the filling factor correction, which does not show the spectral increase towards the inner Galaxy caused by thermal absorption.

\subsubsection{Remarks}

The NE2001 model represents the average large-scale electron density distribution, including some sources and large complexes like the Gum nebula. However, some similarly prominent features like the Cygnus complex at about $l \sim 90^{\circ}$ are missing as it is clearly seen in the free-free emission template and the low-frequency absorption spectra. This seems to be related to the much larger number of pulsars in the southern sky, which allows a modelling of such features there. After considering appropriate filling factors the NE2001 model is able to reproduce the WMAP free-free emission template at $22.8 \mathrm{GHz}$ and also the low-frequency absorption quite well.

In summary the NE2001 model combined with the filling factor obtained by Berkhuijsen et al. (2006) are proven to be sufficient to model the low latitude DIG. We will discuss later that there are observational problems suggesting that at highlatitudes the thermal electrons density is underestimated.

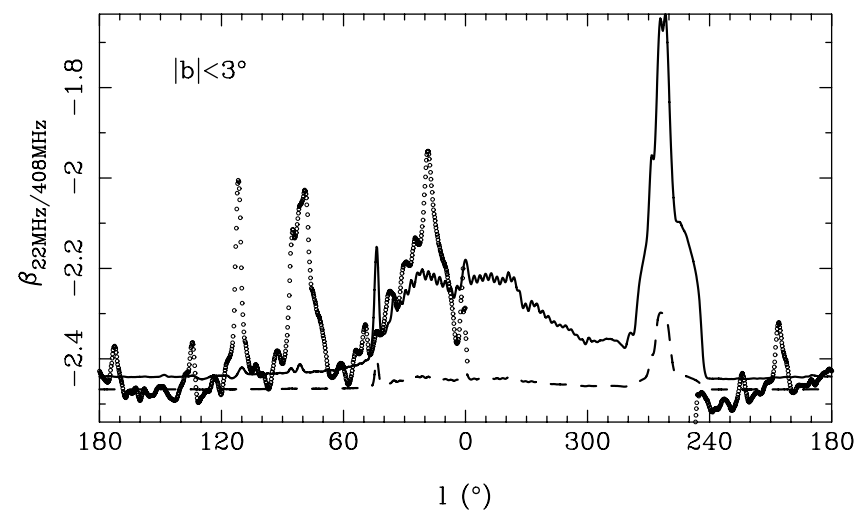

Fig. 8. The average spectral index along the Galactic plane $\left(|b|<3^{\circ}\right)$ calculated from two simulated maps at $408 \mathrm{MHz}$ and $22 \mathrm{MHz}$ including thermal absorption from the NE2001 model with (solid line) and without a filling factor correction (dashed line). For comparison we show the observed spectral indices $\left(|b|<3^{\circ}\right)$ calculated from the $22 \mathrm{MHz}$ northern sky survey map (Roger et al. 1999) and the $408 \mathrm{MHz}$ map (Haslam et al. 1982) at an angular resolution of $2^{\circ}$ (circles).

\subsection{Regular magnetic field properties}

The Galactic magnetic field is commonly described as a vector with a random component $\boldsymbol{b}$ and a regular component $\boldsymbol{B}$. It can be written as, $\boldsymbol{B}_{\text {tot }}=\boldsymbol{B}+\boldsymbol{b}$. Following the procedure described in Sect. 3, the random field component will be discussed together with the $\mathrm{CR}$ electron distribution. The regular field properties are constrained by RMs. Below we use $B=|\boldsymbol{B}|$ to denote the strength of the magnetic field vector. We treat the regular field component as the combination of a disk field $\boldsymbol{B}^{\mathrm{D}}$ and a halo field component $\boldsymbol{B}^{\mathrm{H}}$. The former is constrained by RMs near the Galactic plane $\left(|b|<5^{\circ}\right)$ and the latter by an all-sky RM map from EGS data.

\subsubsection{The disk field}

It is generally accepted that our Galaxy has an organized largescale disk field similar to other nearby Galaxies (see reviews by e.g. Han \& Wielebinski 2002). As described by Beck et al. (1996) the field more or less follows a spiral pattern that can be basically classified into an axi-symmetric spiral (ASS) with no dependence on the azimuthal angle or a bi-symmetric spiral (BSS), which has a symmetry of $\pi$. Relative to the Galactic plane the field above and below can be either symmetric (even parity or quadrupole) or asymmetric (odd parity or dipole).

Magnetic fields are often discussed in the context of propagation of high-energy cosmic rays. The ASS field configuration has been used by Stanev (1997) and the BSS field configuration by Stanev (1997), Tinyakov \& Tkachev (2002) and Prouza \& S̆mída (2003) for modelling. A logarithmic spiral arm field was proposed by Page et al. (2007) to interpret the WMAP observations. We collected a number of published models and checked them for their ability to simulate the observed RMs in the Galactic plane. The results are shown in Fig. 9. Obviously none of the models is able to reproduce the observed systematic features. This motivates us to revise the parameters previously used. 

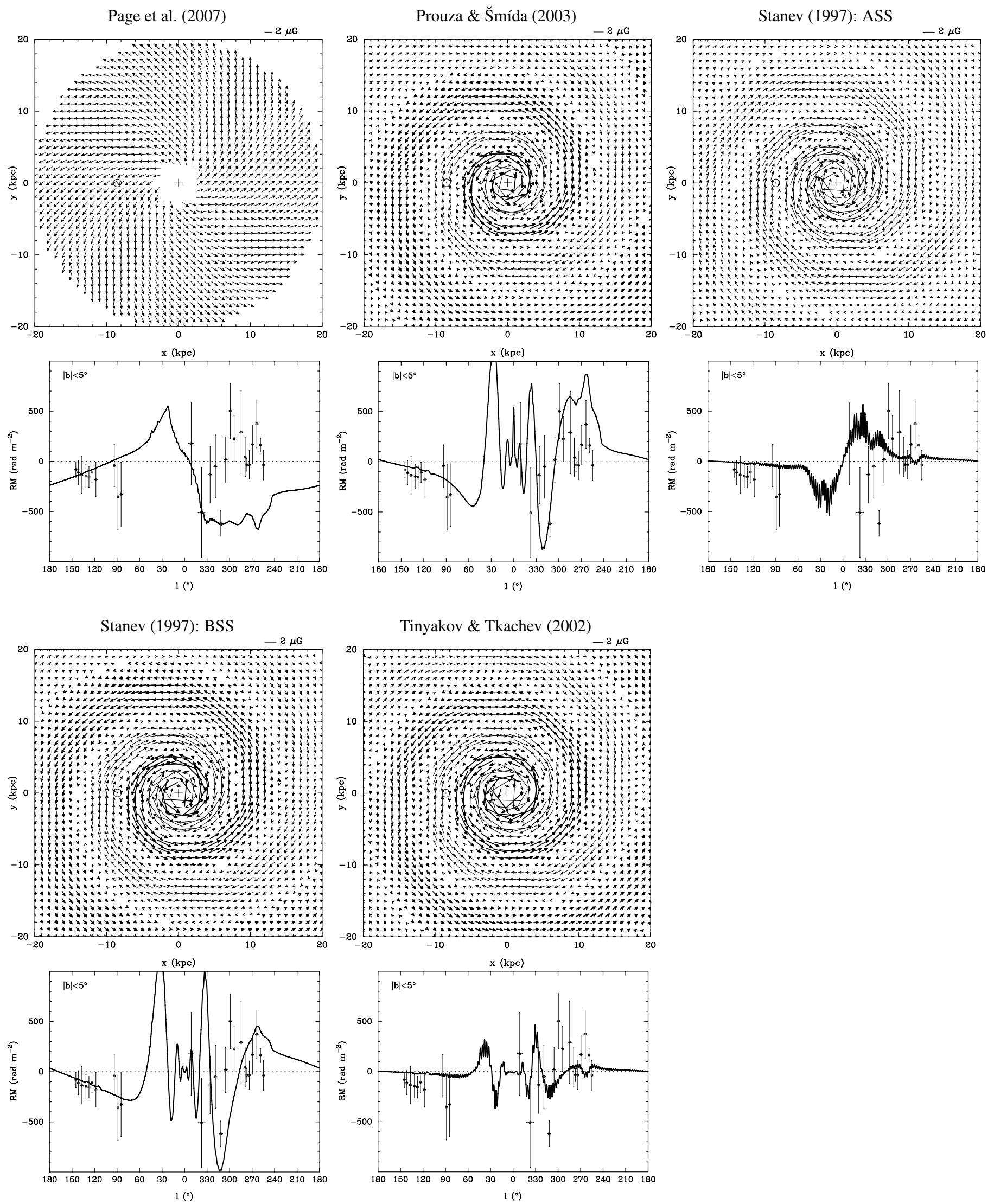

Fig. 9. Different magnetic field configurations in the Galactic plane as seen from the north Galactic pole. The corresponding RM profiles along the Galactic plane $\left(|b|<5^{\circ}\right)$ are shown in the panel below. The average of RMs within longitude bins from the CGPS and the SGPS are shown for comparison. 

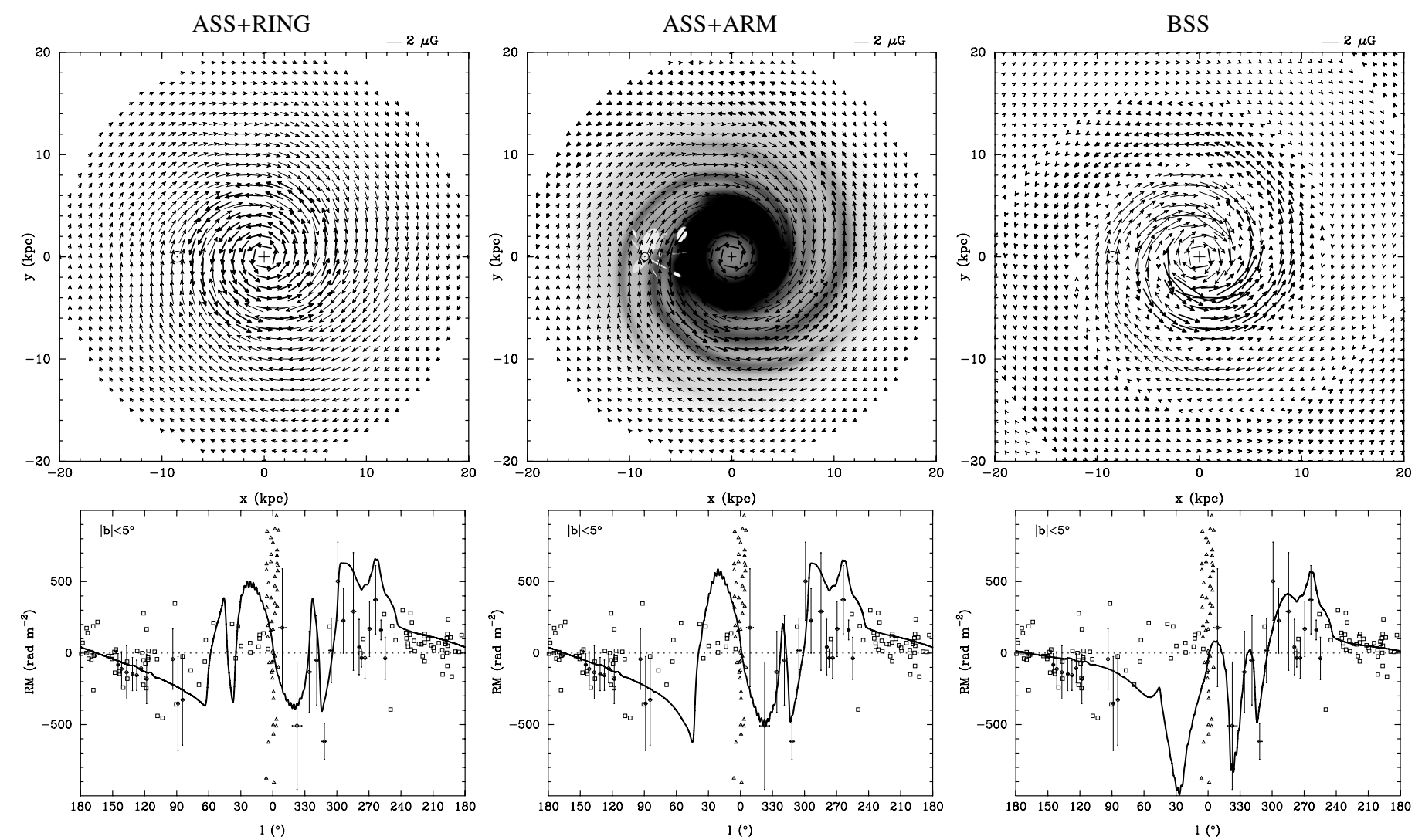

Fig. 10. The same as Fig. 9, but for the ASS+RING, the ASS+ARM and the BSS field configurations. Beside the binned CGPS and SGPS data the RMs near the center from Roy et al. (2005) and RMs from the compilation of Han et al. (1999) are shown by triangles and squares, respectively. For the ASS+ARM model the NE2001 model is overlaid onto the field in gray scale.

The general form of a coplanar and constant pitch angle spiral magnetic field configuration can be written in the cylindrical coordinate system as

$\left\{\begin{array}{l}B_{R}^{D}=D_{1}(R, \phi, z) D_{2}(R, \phi, z) \sin p \\ B_{\phi}^{D}=-D_{1}(R, \phi, z) D_{2}(R, \phi, z) \cos p \\ B_{z}^{D}=0\end{array}\right.$

where $D_{1}(R, \phi, z)$ constrains the spatial variation of the field strength and $D_{2}(R, \phi, z)$ introduces reversals or assymmetries. The definition of the pitch angle $p$ is the same as previously used by Han \& Qiao (1994). We use the function $D_{1}(R, \phi, z)$ always in the following way:

$D_{1}(R, z)=\left\{\begin{array}{cc}B_{0} \exp \left(-\frac{R-R_{\odot}}{R_{0}}-\frac{|z|}{z_{0}}\right) & R>R_{\mathrm{c}} \\ B_{\mathrm{c}} & R \leq R_{\mathrm{c}} .\end{array}\right.$

However, for different models the variables $R_{0}, R_{\mathrm{c}}$ and $z_{0}$ may change. This kind of variation is the same as used by Han et al. (2006) to describe the magnetic field from pulsar RM observations. Too few RM data were observed so far in the first quadrant, which does not allow a detailed modelling of the field within the inner Galaxy. Therefore we keep the field strength constant there.

The regular $z$-component of the Galactic magnetic field is very small. Its strength is about $0.2 \mu \mathrm{G}$ to $0.3 \mu \mathrm{G}$ as estimated by Han \& Qiao (1994). We assume $B_{z}^{D}$ to be zero in our models.

Below we list three different magnetic field models: (1) the ASS model plus reversals in rings (ASS+RING);
(2) the ASS model plus reversals following arms (ASS+ARM); and (3) the BSS model. Following the procedure described in Sect. 3 we use the NE2001 model for the thermal electrons to adjust the parameters in the three models by a trial-and-error approach in order to achieve the best fit for the observed RMs in the plane. The results are summarized below:

$A S S+R I N G$ - In this model, the parameters in $D_{1}(R, z)$ are $R_{0}=10 \mathrm{kpc}, z_{0}=1 \mathrm{kpc}, R_{\mathrm{c}}=5 \mathrm{kpc}, B_{0}=2 \mu \mathrm{G}$ and $B_{\mathrm{c}}=$ $2 \mu \mathrm{G}$. The field reversals are specified by $D_{2}(R)$ being symmetric in $z$ as,

$$
D_{2}(R)= \begin{cases}+1 & R>7.5 \mathrm{kpc} \\ -1 & 6 \mathrm{kpc}<R \leq 7.5 \mathrm{kpc} \\ +1 & 5 \mathrm{kpc}<R \leq 6 \mathrm{kpc} \\ -1 & R \leq 5 \mathrm{kpc}\end{cases}
$$

Here +1 means a clockwise direction as seen from the north pole. The pitch angle is taken as $-12^{\circ}$, which is the average of pitch angles used for the spiral arms in the NE2001 model.

It can be seen from Fig. 10 that the simulated RMs in the Galactic plane fit the data of the CGPS and the SGPS well. We also used the RM data from Roy et al. (2005) near the centre and elsewhere from the compilation of Han et al. (1999). Although the number of measurements is small they basically support the results from the simulations.

$A S S+A R M$ - In this model the parameters for $D_{1}(R, z)$ are $R_{0}=8.5 \mathrm{kpc}, z_{0}=1 \mathrm{kpc}, R_{\mathrm{c}}=5.3 \mathrm{kpc}, B_{0}=2 \mu \mathrm{G}$ and $B_{\mathrm{c}}=2 \mu \mathrm{G}$. We use similar logarithmic arms as Cordes \& Lazio (2003) (see Fig. 5), but with a pitch angle of $-12^{\circ}$. The pitch angle of the magnetic field is the same as that of the arms. The 


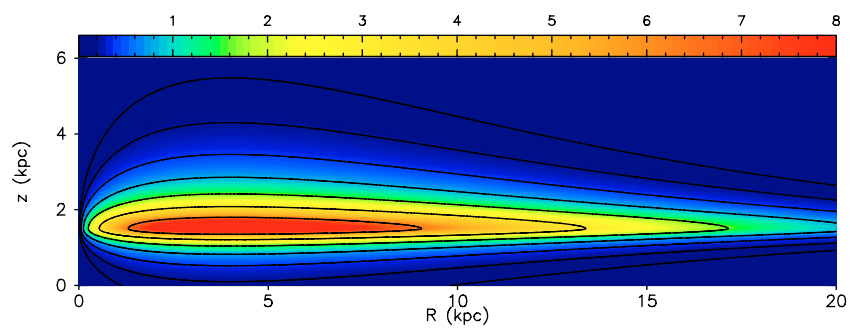

Fig. 11. The strength of the regular halo magnetic field component. Contours start at $0.1 \mu \mathrm{G}$ and increase by a factor of 2 . The field below the plane has the same strength but is reversed.

reversals are placed between the inner edge of the SagittariusCarina arm and the inner edge of the Scutum-Crux arm. This reversal is consistent with that found by Brown et al. (2007) based on the SGPS RM data. However, this should not be taken as a final conclusion because the amount of RM data is small and also the position and the extent of the spiral arms is uncertain. As can be seen from Fig. 10 this model fits the observations as well as the ASS+RING model.

$B S S$ - In this model the parameters for $D_{1}(R, z)$ are $R_{0}=$ $6 \mathrm{kpc}, z_{0}=1 \mathrm{kpc}, R_{\mathrm{c}}=3 \mathrm{kpc}, B_{0}=2 \mu \mathrm{G}$ and $B_{\mathrm{c}}=2 \mu \mathrm{G}$. $D_{2}(R, \phi)$ is written as

$D_{2}(R, \phi)=-\cos \left(\phi+\beta \ln \frac{R}{R_{b}}\right)$

where $\beta=1 / \tan p$. The parameters are $R_{b}=9 \mathrm{kpc}$ and $p=-10^{\circ}$ for $R>6 \mathrm{kpc}$ and otherwise $R_{b}=6 \mathrm{kpc}$ and $p=-15^{\circ}$. The model is able to fit the RM data from the SGPS and the CGPS (Fig. 10), but is inconsistent with other data as discussed below.

\subsubsection{The halo field}

The synthesized all-sky RM map (Fig. 3) shows an asymmetry in longitude and latitude relative to the Galactic plane and the Galactic centre, respectively, indicating an asymmetric halo magnetic field configuration.

Such a RM configuration could in principle also originate from large local shells. However, at least two shells with opposite magnetic field orientations located below and above the plane are needed. Han et al. (1999) showed that the RMs of pulsars with distances larger than $1 \mathrm{kpc}$ increase with distance, which makes an explanation by local shells unlikely and strongly supports the large-scale field scenario.

We follow Prouza \& S̆mída (2003) for a description of the regular double-torus field component for one half of the Galaxy and the reversed direction in the other half:

$$
B_{\phi}^{\mathrm{H}}(R, z)=B_{0}^{\mathrm{H}} \frac{1}{1+\left(\frac{|z|-z_{0}^{\mathrm{H}}}{z_{1}^{\mathrm{H}}}\right)^{2}} \frac{R}{R_{0}^{\mathrm{H}}} \exp \left(-\frac{R-R_{0}^{\mathrm{H}}}{R_{0}^{\mathrm{H}}}\right) .
$$

The parameters are $z_{0}^{\mathrm{H}}=1.5 \mathrm{kpc}, z_{1}^{\mathrm{H}}=0.2 \mathrm{kpc}$ for $|z|<z_{0}^{\mathrm{H}}$ and otherwise $z_{1}^{\mathrm{H}}=0.4 \mathrm{kpc}, B_{0}^{\mathrm{H}}=10 \mu \mathrm{G}$, and $R_{0}^{\mathrm{H}}=4 \mathrm{kpc}$. The strength of the halo magnetic field above the plane is shown in Fig. 11.

We add the halo field to the disk field, run the simulation and obtain average longitude profiles for $25^{\circ}<b<35^{\circ}$ and $-35^{\circ}<b<-25^{\circ}$ as shown in Fig. 12. Some RMs of EGSs at longitudes of about $90^{\circ}$ and larger show large deviations both above and below the plane. These areas coincide with the positions of Loop II and Loop III. However, whether the Loop
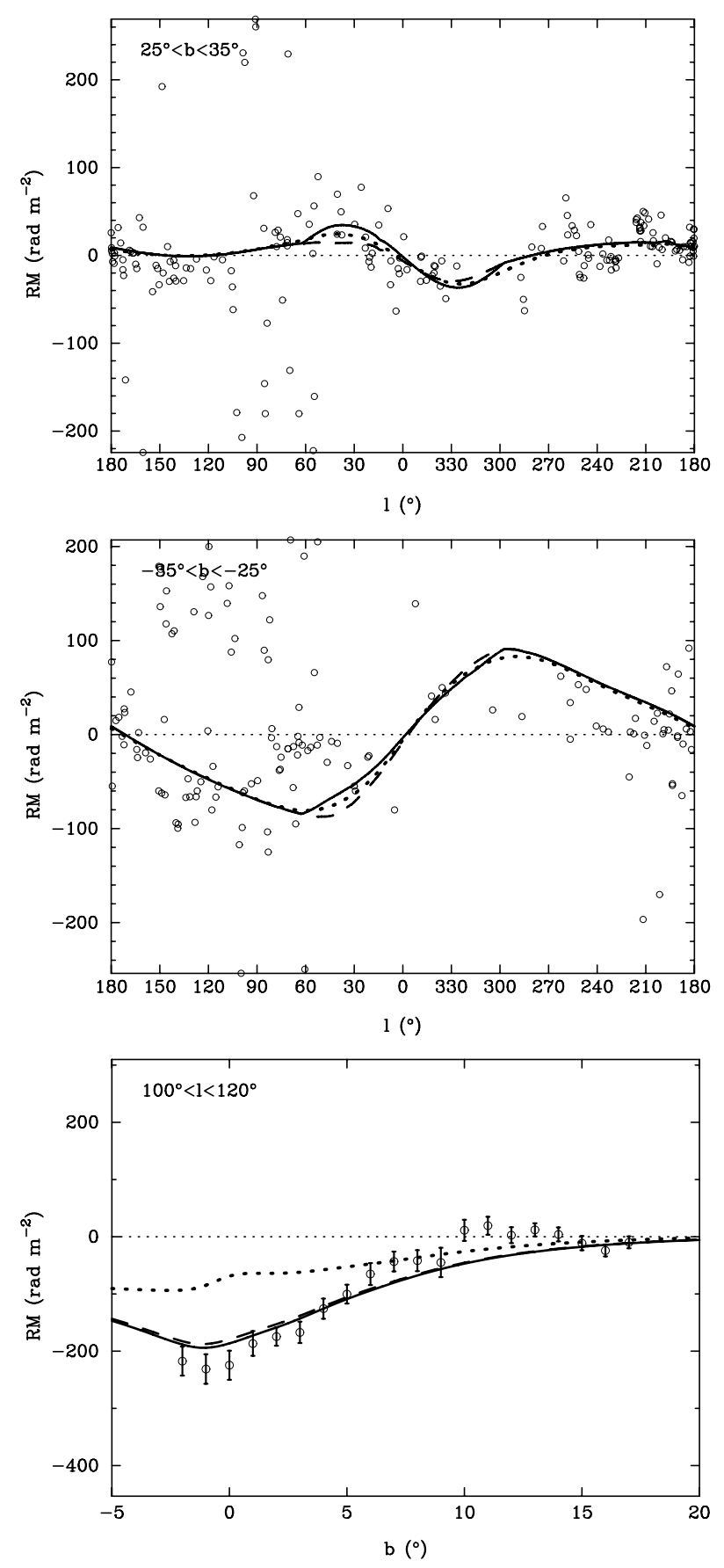

Fig. 12. RM profiles from the halo field plus the ASS+RING model (solid), the ASS+ARM (dashed) model and the BSS model (dotted) versus the observed RM data. The RM data in the bottom panel are from the extended CGPS averaged for latitude intervals of $2^{\circ}$ (see text).

structures physically cause these RM deviations needs a further check. Except for these outliers the simulations are generally consistent with the observational data.

We show the latitude variation of the simulated RMs in Fig. 12 and in addition the average $\mathrm{RM}$ between $l=100^{\circ}$ and $l=120^{\circ}$ versus latitude from $-5^{\circ}$ to $20^{\circ}$. The observed data are from the extended CGPS (Jo-Anne Brown et al., in prep.). The halo field plus the ASS+RING or the ASS+ARM disk fields yield quite a good fit. However, the halo field plus the BSS field is clearly inconsistent with the data. If we tune the BSS field to match the latitude profile, we fail to get a good fit to the RMs 
along the Galactic plane. We conclude that the BSS model is not in agreement with the observed RMs of EGSs.

\subsubsection{Remarks}

We introduced field reversals in our models with sharp transitions, which means an abrupt field change in the opposite direction. This might be not a realistic scenario, however, we do not expect much influence by this simplification as the transition regions are smoothed out in our simulated maps. We also used a constant pitch angle in our models, which is most likely another simplicifation. We note that the ASS+RING and the ASS+ARM models are not very sensitive to the pitch angle used.

\subsection{Random fields, CR electron density and local excess of the synchrotron emission}

\subsubsection{The random magnetic field component}

The knowledge about the properties of random magnetic fields in galaxies is quite limited (Beck 2001). A typical scale for the random fields was claimed to be about $50 \mathrm{pc}$ based on the single cell size model (Rand \& Kulkarni 1989). However, the single cell model is a strong simplification. Structure functions of RMs of EGSs yield the fluctuation properties of $n_{\mathrm{e}} b_{\|}$. Unfortunately, due to the coupling between the electron density and magnetic field the extracting of the magnetic field fluctuation remains very uncertain. Minter \& Spangler (1996) tried to tackle the decoupling by involving the structure function of emission measures and concluded that the magnetic field spectrum can be described by a Kolmogorov spectrum with an outer scale of about $4 \mathrm{pc}$. The turbulence for the region of sky investigated by Minter \& Spangler (1996) occurs in thin sheets or filaments and can be described as a two-dimensional turbulence for scales between 4 pc to about 80 pc. Han et al. (2004) claimed a flatter spectrum for scales between $500 \mathrm{pc}$ and $1.5 \mathrm{kpc}$ based on pulsar RM and DM data.

In principle a stochastic realisation of the random magnetic fields can be generated by the Fourier transform of the square root of the spectrum as (e.g. Martel 2005) $b(s) \propto \int \sqrt{P(\boldsymbol{k})}(\xi+$ i $\zeta) \exp (\mathrm{i} \boldsymbol{k} \cdot \boldsymbol{s}) \mathrm{d} \boldsymbol{s}$, where $P(\boldsymbol{k})$ is the power spectrum, $\boldsymbol{k}$ is the wavenumber, $\boldsymbol{s}$ is the spatial position, and $\xi$ and $\zeta$ are Gaussian random numbers. This requires a 3D inverse Fourier transformation to obtain $b(s)$. To fulfill the above equation the Galaxy must be gridded to small cubic boxes with a volume of $l^{3}$. To avoid an intrinsic correlation by the selected box size, they must be smaller than the outer scale of the magnetic field fluctuations. Let $l$ have a size of $2 \mathrm{pc}$ and the Galaxy volume to be $40 \mathrm{kpc} \times 40 \mathrm{kpc} \times 10 \mathrm{kpc} 2 \times 10^{12}$ boxes for an all-sky calculation are needed, which is far beyond current computer memory capabilities. However, a small patch of the sky can be treated that way, utilizing a huge amount of computing time. A more detailed discussion on how to obtain a random field from a Kolmogorov spectrum for high-angular resolution simulations will be presented in a subsequent paper, where we will simulate high-resolution patches of Galactic emission as they will be observed by LOFAR and the future SKA.

Here we assume that the random field components are homogeneous and follow a Gaussian distribution in strength with an average of zero and a scatter of $\sigma_{b} / \sqrt{3}$ in each dimension. As discussed later the best value for the mean random field strength is $b=3 \mu \mathrm{G}$ throughout the Galaxy.

\subsubsection{CR electron density}

Synchrotron emission in the frequency range from $10 \mathrm{MHz}$ to $40 \mathrm{GHz}$ originates from $\mathrm{CR}$ electrons with energies between $400 \mathrm{MeV}$ and $25 \mathrm{GeV}$ assuming a magnetic field component perpendicular to the line of sight of $4 \mu \mathrm{G}$ (Webber et al. 1980). Local measurements of the density of CRs with energies below several $\mathrm{GeV}$ are very uncertain due to the limited knowledge of the heliospheric modulation (e.g. Strong et al. 2004). For the $\mathrm{CR}$ electrons with energies higher than several $\mathrm{GeV}$, the measurements (e.g. Barwick et al. 1998; Grimani et al. 2002) follow a simple power law. The flux density can be described as $N(\gamma)=C(R, z) \gamma^{p}$ with $p$ of about -3 , where $\gamma$ is the Lorentz factor. The CR flux density at $10 \mathrm{GeV}$ from the aforementioned measurements is about $0.2\left(\mathrm{GeV} \mathrm{m}^{2} \mathrm{sr} \mathrm{s}\right)^{-1}$ which means $C(R=8.5 \mathrm{kpc}, z=0) \equiv C_{0} \approx 3.2 \times 10^{-5} \mathrm{~cm}^{-3}$. However, it is questionable wheather the local measurements are representative for the CR density elsewhere in the Galaxy (Pohl \& Esposito 1998; Strong et al. 2004). Therefore we regard below the measured local CR flux density as weakly constrained.

We assume that the CR electron distribution consists of a disk component, whose spatial distribution is written as

$C(R, z)=C_{0} \exp \left(-\frac{R-R_{\odot}}{8 \mathrm{kpc}}-\frac{|z|}{1 \mathrm{kpc}}\right)$

with $C_{0}=6.4 \times 10^{-5} \mathrm{~cm}^{-3}$. For the Galactic centre region the CR electron flux density is taken as a constant $C(R<3 \mathrm{kpc})=$ $C(R=3 \mathrm{kpc})$. The disk component is set to zero for $z>1 \mathrm{kpc}$ as discussed in Sect. 7.2.

The spectral index of the CR electrons $p$, the radio flux density $\alpha$ and the brightness temperature $\beta$ are related to each other as $\alpha=(p+1) / 2=\beta+2$. Spectral index maps show spatial variations of the spectral index and a spectral steepening at high frequencies is indicated (Reich et al. 2004). In this paper we simplify the problem and keep the energy spectral index of the CR electrons $p$ constant with a value of $p=-3$ corresponding to $\beta=-3$. For the frequency range below $408 \mathrm{MHz}$ we assume $p$ to be -2 or $\beta=-2.5$.

\subsubsection{The local excess of the synchrotron emission}

The $408 \mathrm{MHz}$ total intensity map shows a nearly constant highlatitude $\left(|b|>30^{\circ}\right)$ emission, which can be explained in two ways. There might exist an isotropic disk-centered halo emission component, which is difficult to model from our location in the Galactic plane far from the centre. However, the halo magnetic field we derived from RMs makes a smooth total intensity halo emission component quite unlikely. Otherwise the highlatitude emission can be attributed to local emission. In fact the observed local synchrotron enhancement requires a local excess of the CR electron density and/or an increase of the local random magnetic field component.

Absorption measurements towards distant HII regions located in the inner Galaxy at $74 \mathrm{MHz}$ yield a synchrotron emissivity of $0.35 \mathrm{~K} \mathrm{pc}^{-1}$ to $1.0 \mathrm{~K} \mathrm{pc}^{-1}$ for distances larger than about $2 \mathrm{kpc}$ (Nord et al. 2006), which is consistent with the $408 \mathrm{MHz}$ emissivity from the model of Beuermann et al. (1985) for a typical spectral index $\beta=-2.5$ for low frequency synchrotron emission (Roger et al. 1999). The $22 \mathrm{MHz}$ survey absorption data from HII regions by Roger et al. (1999) give an emissivity of about $30 \mathrm{~K} \mathrm{pc}^{-1}$ for distances less than about $1 \mathrm{kpc}$, which is a factor of about 2 larger than the typical emissivities obtained by Beuermann et al. (1985), indicating a local synchrotron enhancement by a factor of 2. Fleishman \& Tokarev (1995) 

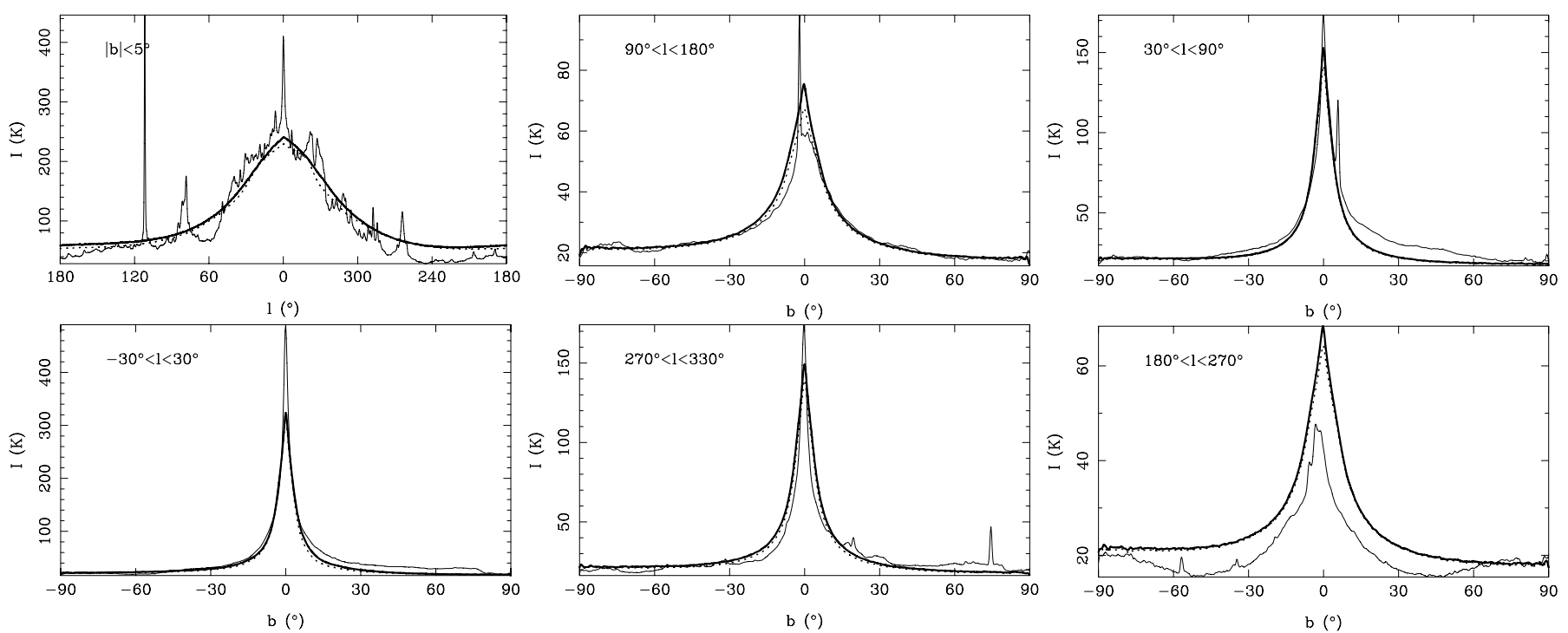

Fig. 13. Total intensity profiles at $408 \mathrm{MHz}$ for three magnetic field models. The thin solid lines stand for the profiles from the $408 \mathrm{MHz}$ all sky survey. The results from the ASS+RING (thick solid lines), the ASS+ARM (dashed) and the BSS (dotted) disk field models are also shown. The simulated maps have been smoothed to an angular resolution of $51^{\prime}$.
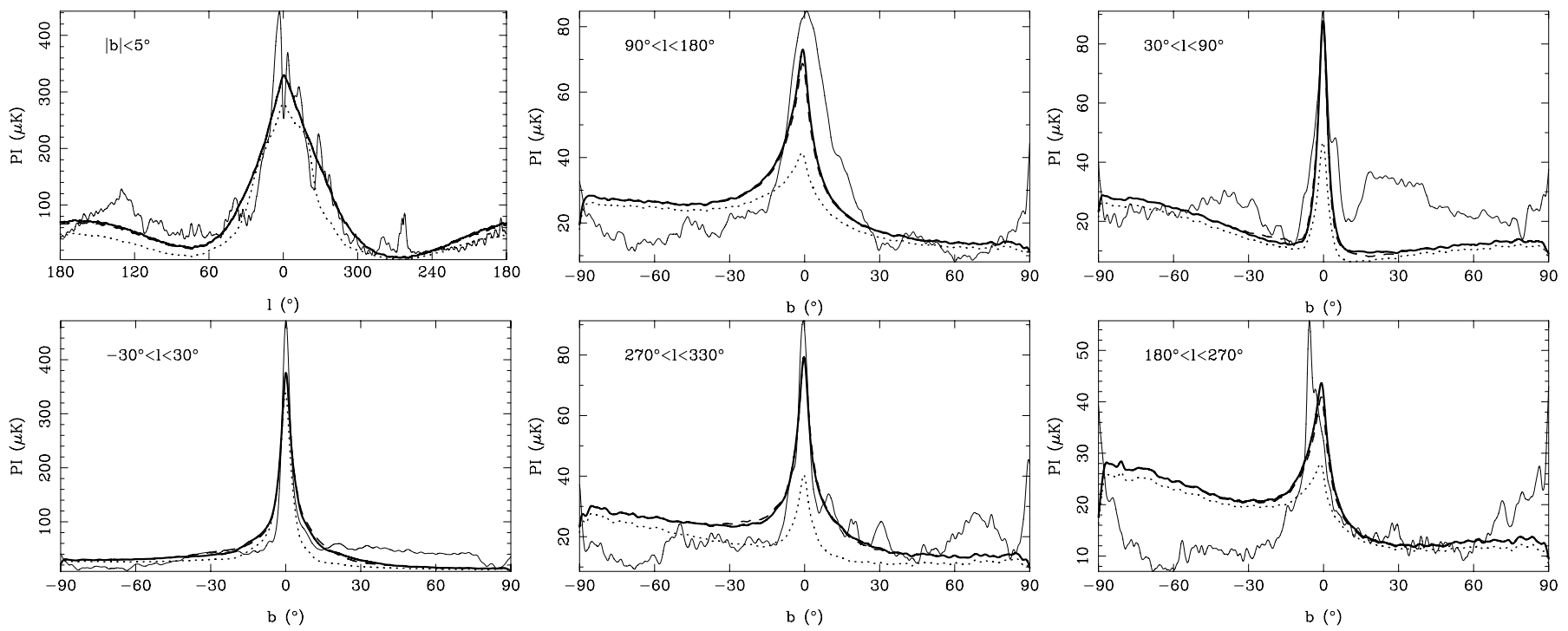

Fig. 14. The same as Fig. 13, but for profiles of polarized intensity at $22.8 \mathrm{GHz}$. All the maps are smoothed to an angular resolution of $2^{\circ}$.

summarized low-frequency absorption measurements up to $2 \mathrm{kpc}$, which indicate a local synchrotron enhancement by a factor of about 3. From polarization modelling towards the very local Taurus molecular cloud Wolleben \& Reich (2004) confirmed a significant local synchrotron excess.

The solar system is located in a low-density region filled with hot gas, called the "local bubble". Its size is about $200 \mathrm{pc}$. The "local bubble" is believed to be created by about twenty supernovae from the nearby Scorpius-Centaurus OB clusters during the last 10-15 Myr. The last explosion may have occurred about 1 Myr ago (Breitschwerdt \& de Avillez 2006; Fuchs et al. 2006). There is compelling evidence that shock acceleration in SNRs is the source for energetic CR electrons (e.g. Kobayashi et al. 2004). With a typical diffusion coefficient of $10^{28} \mathrm{~cm}^{2} \mathrm{~s}^{-1}$ (Kobayashi et al. 2004) CR electrons may have reached about 600 pc distance and thus increase the CR electron density in the local bubble. Alternatively, multiple fragments with compressed magnetic fields from the shells of the numerous very old SNRs forming the "local bubble" might have survived in the interstellar medium and enhance the local synchrotron emission. From this scenario the local excess of the synchrotron emission can be achieved either by an increase of the local CR electron density and/or by an enhanced local random magnetic field as already stated earlier. We have tested both cases and find that the results are the same. For the simulation results shown below we assume a local spherical component within $1 \mathrm{kpc}$ from the Sun, where the CR electron density exceeds the disk component by a factor 1.5 .

\subsubsection{Simulated $408 \mathrm{MHz}$ total intensity and $22.8 \mathrm{GHz}$ polarized intensity}

In Figs. 13 and 14 the latitude and longitude profiles from the simulated maps are compared with those from the $408 \mathrm{MHz}$ and the WMAP $22.8 \mathrm{GHz}$ polarized intensity all-sky surveys. All three models fit the total intensity profiles quite well. However, 
for polarized intensities the BSS magnetic disk field cannot well reproduce the small longitude asymmetry relative to the Galactic centre along the Galactic plane (Fig. 14).

The direction of the halo magnetic field is in the opposite direction to the disk field above and in the same direction below the Galactic plane. Therefore the resulting total magnetic field strength is stronger below than above the Galactic plane. In our model the CR electron density is truncated at $z=1 \mathrm{kpc}$ and the halo field decreases quickly towards the Galactic plane, which makes the resulting intensity difference quite small, e.g. about $20 \mu \mathrm{K}$ for polarized $22.8 \mathrm{GHz}$ emission (see Fig. 14). Therefore this asymmetry is difficult to prove observationally and requires a careful absolute zero-level setting for the large-scale emission. For the case of a smaller halo magnetic field and an extent of the $\mathrm{CR}$ electrons beyond the present limit of $1 \mathrm{kpc}$ in $z$ this difference will remain small.

\subsubsection{Remarks on the random Galactic magnetic field}

We assume a random field component of uniform strength throughout the Galaxy. However, the random magnetic field could be considered to be stronger in the Galactic plane, where supernova explosions and violent star formation processes inject energy by shock waves or stellar winds into the interstellar medium and thus initiate higher fluctuations of the magnetic field than at high latitudes. From the analysis of the structure function of EGSs it was shown that fluctuations of the magnetized interstellar medium in the Galactic plane are longitude-dependent (Sun \& Han 2004) and also different for arm and inter-arm regions (Haverkorn et al. 2006). Our actual simulations are not sensitive to spatial variations of the random magnetic field at high-latitudes because of the present truncation of the CR electrons at $z=1 \mathrm{kpc}$.

The local total magnetic field vector $\boldsymbol{B}_{\text {tot }}=\boldsymbol{B}+\boldsymbol{b}$ in our model is about $4 \mu \mathrm{G}$ with a ratio between the random to the regular magnetic field component of about 1.5. We note that this total field strength is just consistent with the value of $6 \pm 2 \mu \mathrm{G}$, which was derived by assuming energy equipartition between CRs and magnetic fields from the synchrotron model of Beuermann et al. (1985) (Berkhuijsen, personal communication, shown in Fig. 1 in Beck 2001).

\subsection{Depolarization}

Using the Galactic magnetic field model and the CR electron distribution we are able to model the observed total intensity map at $408 \mathrm{MHz}$ and the polarized intensity map at $22.8 \mathrm{GHz}$. The $1.4 \mathrm{GHz}$ total intensity map has a different amount of thermal and non-thermal components and is also well modelled (not shown here). The $1.4 \mathrm{GHz}$ polarized intensity originates from synchrotron emission modulated by the interstellar medium and should be reproduced as well after accounting for depolarization effects, which needs to be discussed in some detail.

\subsubsection{Simulation problems}

We show the simulated results for polarized intensity at $1.4 \mathrm{GHz}$ in Fig. 15, where the longitude profiles along the Galactic plane and the latitude profiles for $|l|<30^{\circ}$ are presented. The observed profiles show strong depolarization observed in particular towards the inner Galaxy (see Fig. 2). Note that prominent large structures like the "Fan region" and Loop I (as indicated in Fig. 15) are not included in our modelling, as they are
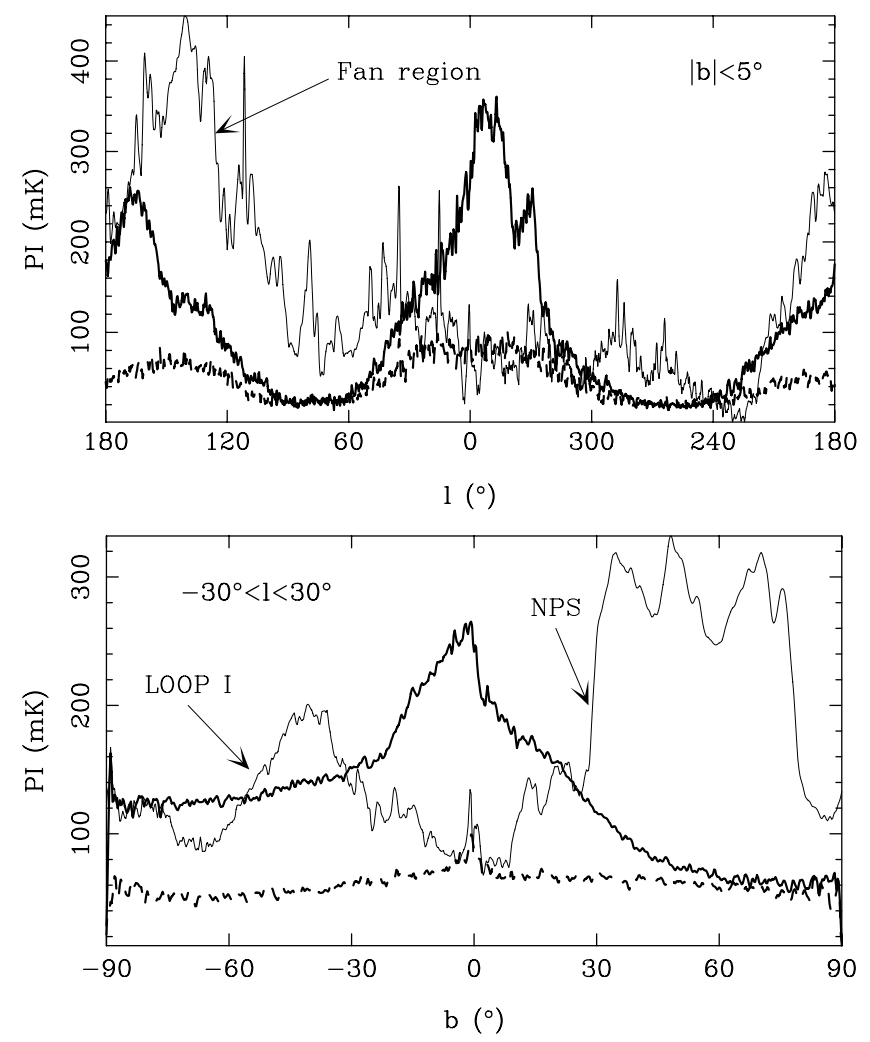

Fig. 15. Simulated polarized intensities at $1.4 \mathrm{GHz}$ (shown as thick solid and dashed lines) along Galactic longitude in the plane (upper panel) and along Galactic latitude (lower panel) are shown in comparison to the $1.4 \mathrm{GHz}$ observations (thin solid lines). The simulation shown in dashed lines includes a correction for RM fluctuations based on the thermal electron filling factor and a related magnetic field enhancement. For details see text. The angular resolution is always $36^{\prime}$.

considered as local structures. Therefore the lower envelopes of the observed profiles have to be compared with the simulations. Towards longitudes $l= \pm 90^{\circ}$ the magnetic field direction is almost tangential to the line of sight, which makes any polarized intensity intrinsically small and the simulated polarized intensities at $1.4 \mathrm{GHz}$ are almost consistent with the observed ones. However, towards the centre and the anti-centre region the simulated polarized intensity is in general much larger than that observed.

We show in the following that the random magnetic field component is properly set in our simulation: assuming that the intrinsic random field component is located in cells with a common size $l$, the number of cells in the $i$ th volume unit calculates then as $N_{\mathrm{c}}^{i}=i^{2} \Delta r^{3} \Delta \theta^{2} / l^{3}$. In the outer (large $i$ ) unit the number of cells is very large. However, in the simulation we specify also for the outer unit just one direction for the random magnetic field component, which means the number of random magnetic field cells is clearly underestimated. This way the simulated polarized emission may be too large. If this is the case the simulated polarized emission should significantly decrease when the maps are smoothed. We smoothed the simulated polarization map to an angular resolution of $36^{\prime}$, which is the resolution of the observed $1.4 \mathrm{GHz}$ all-sky map, and to a resolution of $2^{\circ}$. The results are shown in Fig. 16 for a cut along the Galactic plane. It is evident that the changes in the profile after smoothing are small, which indicates that the cell size for the random field component is small enough even for large radii. This proves that the random field component to be properly considered in the simulations. 


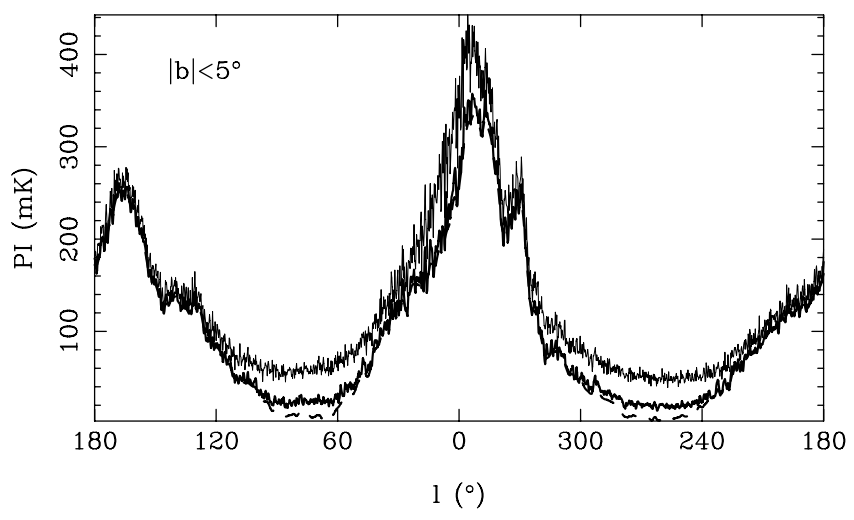

Fig. 16. Simulated polarized intensities at $1.4 \mathrm{GHz}$ along the Galactic plane are shown at $15^{\prime}$ (thin solid line) and after smoothing to $36^{\prime}$ (thick solid line) and $2^{\circ}$ (dashed line).

\subsubsection{Solutions to increase the depolarization}

As shown by Sokoloff et al. (1998) Faraday depolarization effects become strong when either the RM or the RM fluctuations along the line of sight $\left(\sigma_{\mathrm{RM}}\right)$ increases. For our simulations the RM fluctuations can be estimated: $\sigma_{\mathrm{RM}} \approx 0.81\left\langle n_{\mathrm{e}}\right\rangle \sigma_{b} \sqrt{r \Delta r}$, where $\left\langle n_{\mathrm{e}}\right\rangle$ is the average thermal electron density. In our calculation $\Delta r=20 \mathrm{pc}$. For typical values such as $r=10 \mathrm{kpc}$, $\left\langle n_{\mathrm{e}}\right\rangle=0.03 \mathrm{~cm}^{-3}, \sigma_{b}=3 \mu \mathrm{G}$, the RM fluctuation can be estimated to be about $32 \mathrm{rad} \mathrm{m}^{-2}$. This fluctuation should produce enough depolarization at $1.4 \mathrm{GHz}$ based on the formula by Sokoloff et al. (1998). However, the assumption of identical distributions of all constituents of the magnetic-ionic medium along the line of sight made by Sokoloff et al. (1998) clearly differs from the more realistic properties used for our simulation. The observed depolarization at $1.4 \mathrm{GHz}$ suggests that this $\mathrm{RM}$ fluctuation is too small to cause significant depolarization.

The mean RM has already been constrained by extragalactic sources. We try to increase the RM fluctuations in each volume unit to introduce a larger depolarization. We rewrite the formula for RM fluctuations for the case of a clumpy thermal medium using the same units as in Eq. (1), which reads:

$\sigma_{\mathrm{RM}}^{2}=0.81^{2}\left\langle n_{\mathrm{e}}^{2} b^{2}\right\rangle r \Delta r=0.81^{2} \frac{\left\langle n_{\mathrm{e}}\right\rangle^{2} \sigma_{b}^{2}}{f_{\mathrm{nb}}} r \Delta r$,

where the factor $f_{\mathrm{nb}}$ is defined as

$f_{\mathrm{nb}} \equiv \frac{\left\langle n_{\mathrm{e}}\right\rangle^{2} \sigma_{b}^{2}}{\left\langle n_{\mathrm{e}}^{2} b^{2}\right\rangle}$

Then we calculate the RMs in each volume unit as $\mathrm{RM}=\mathrm{RM}_{0}+$ $\mathrm{RM}_{r} / \sqrt{f_{\mathrm{nb}}}$, where $\mathrm{RM}_{0}$ and $\mathrm{RM}_{r}$ are calculated from the regular and random magnetic field components, respectively. From the simulations we found that for $f_{\mathrm{nb}}=0.01$ the polarized intensity in the Galactic plane is significantly reduced and the simulated results are consistent with the observations, as seen in Fig. 15. The correction does not change the total RM along the line of sight on average, which we prove in Fig. 17, because just the random RM component in each volume unit is increased.

What is the physical meaning of the factor $f_{\mathrm{nb}}$ ? If the thermal electron density and the random field are independent, i.e., $\left\langle n_{\mathrm{e}}^{2} b^{2}\right\rangle=\left\langle n_{\mathrm{e}}^{2}\right\rangle\left\langle b^{2}\right\rangle$, the factor $f_{\mathrm{nb}}$ is identical to the thermal electron filling factor $f_{\mathrm{e}}$. Note that $\left\langle b^{2}\right\rangle=\sigma_{b}^{2}$. The thermal electron filling factor in the plane is about 0.07 from our simulations, while Berkhuijsen et al. (2006) derived values for $f_{\mathrm{e}}$ between 0.04 and 0.07 , which is larger than the value $f_{\mathrm{nb}}=0.01$

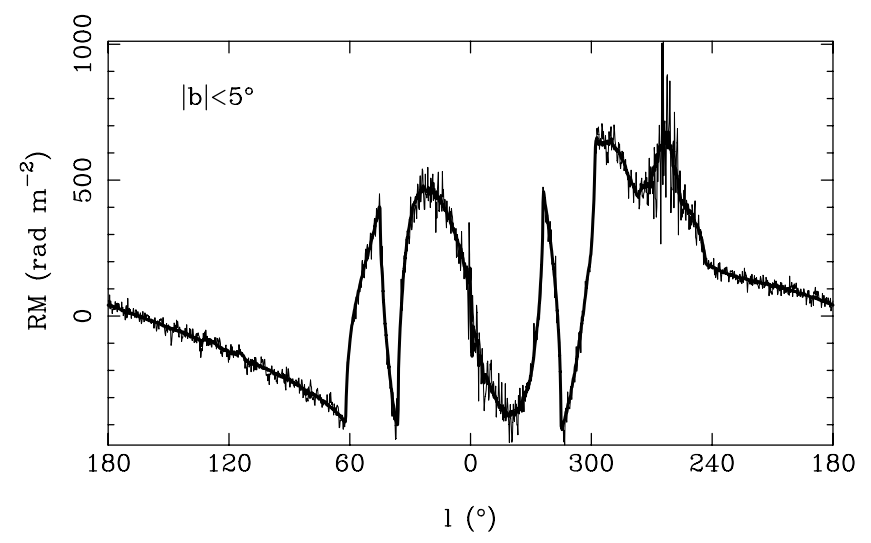

Fig. 17. The original simulated RM distribution for the ASS+RING disk field (thin line) and the RM distribution including RM fluctuations by $f_{\mathrm{nb}}=0.01$ (solid line).

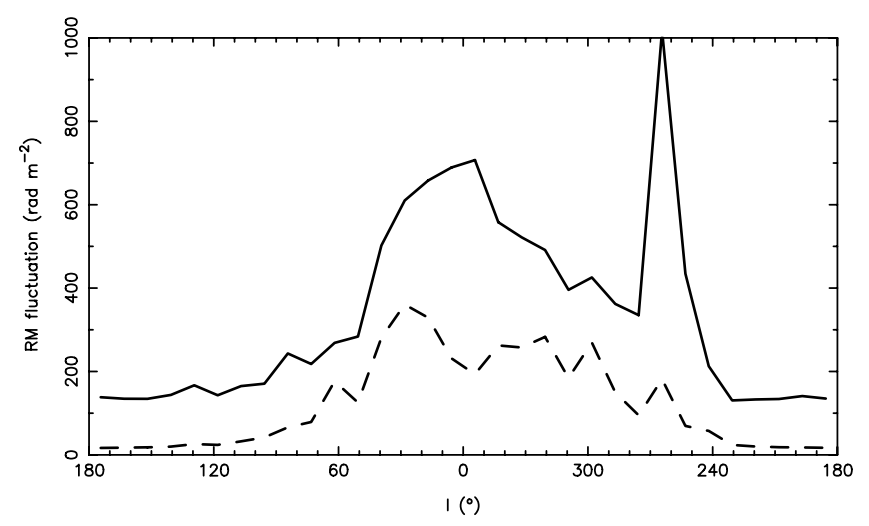

Fig. 18. The spatial fluctuations of RMs before (dashed) and after (solid line) considering $\mathrm{RM}$ variations $\left(f_{\mathrm{nb}}=0.01\right)$ in a clumpy magnetized WIM along the line of sight.

as obtained above. This indicates a coupling between the thermal electron density and the random magnetic field strength. In general we may write $f_{\mathrm{nb}}=f_{\mathrm{e}} f_{\mathrm{c}}$, where we take $f_{\mathrm{c}}$ as the coupling factor. In case a dependence like $b^{2} \propto\left(n_{\mathrm{e}}^{2}\right)^{\alpha}$ exists, we have $f_{\mathrm{c}} \propto f_{\mathrm{e}}^{\alpha}$. Our simulations clearly favor $f_{\mathrm{nb}}<f_{\mathrm{e}}$ with $\alpha>0$. This means that the thermal electron density and the random magnetic field strength are correlated. However, we note that the physics and a theoretical value for the coupling factor needs to be worked out. Therefore we take our results as qualitative and did not attempt to account for possible spatial variations of $f_{\mathrm{nb}}$ as it is suggested by $f_{\mathrm{e}}(z)$.

\subsubsection{Comment on the observed RM fluctuations}

We finally discuss the observed large fluctuating RMs from EGSs in the Galactic plane (see Fig. 4). Note that these fluctuations are in principle different from those we discussed above. RM variations of EGSs result from different ISM properties for each line of sight, while RM fluctuations causing depolarization are along a single line of sight. However, larger RM variations are expected for different line of sights, if the RM fluctuations are large at all. Therefore both cases are related. As shown in Fig. 18 the spatial RM fluctuations are significantly larger after including the factor $f_{\mathrm{nb}}=0.01$. We note that the large fluctuations are now consistent with the RM observations presented in Fig. 4. An important implication is that the large spatial RM scattering is not due to fluctuations of large random field 

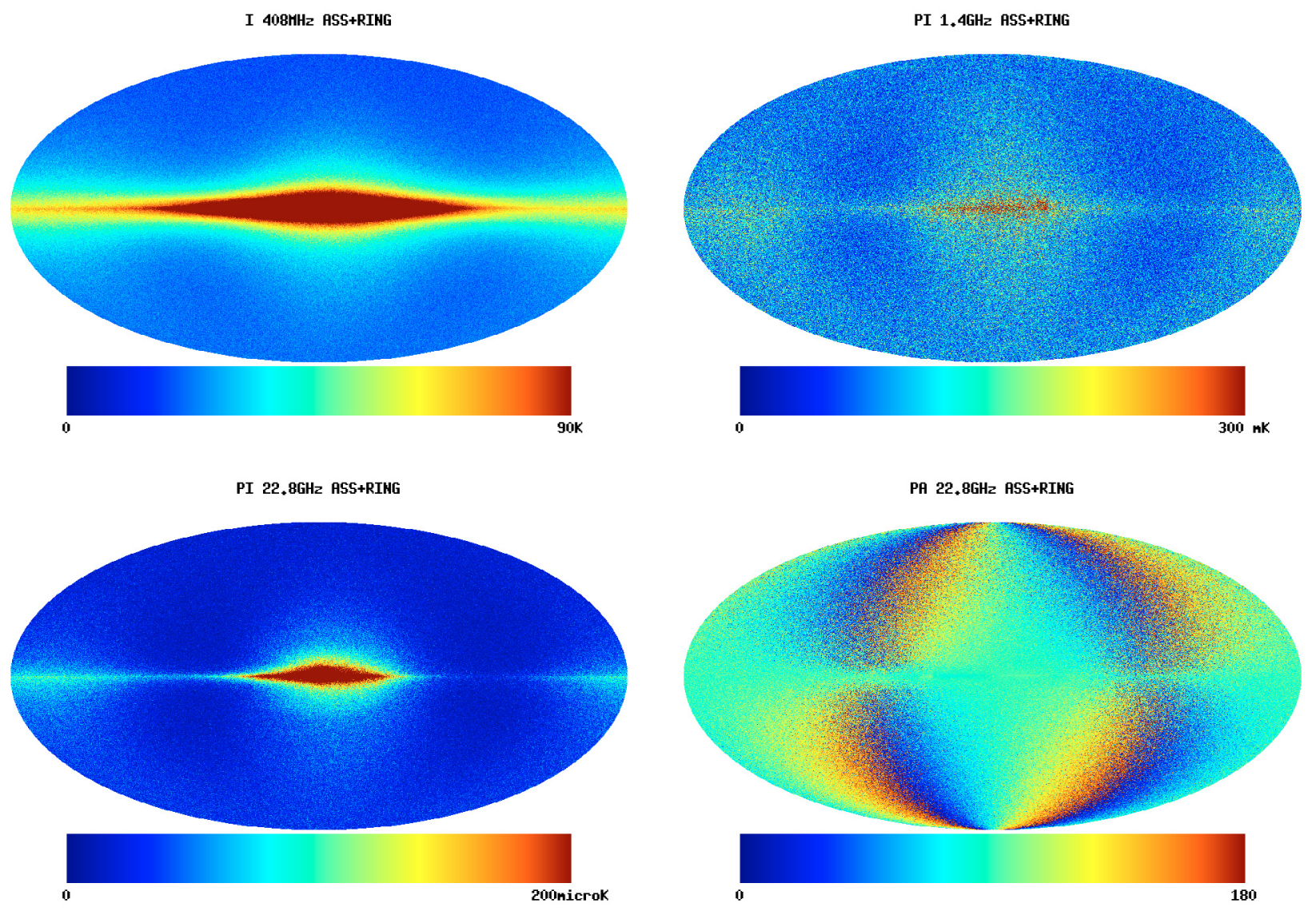

Fig. 19. Simulated all-sky maps for total intensity at $408 \mathrm{MHz}$, polarized intensity at $1.4 \mathrm{GHz}$ and at $22.8 \mathrm{GHz}$ and polarization angle at $22.8 \mathrm{GHz}$ are shown for the ASS+RING magnetic field configuration. All the maps have a resolution of $15^{\prime}$.

components, but to the thermal filling factor and a coupling factor between the electron density and the random field strength of the clumpy magnetized WIM.

\subsection{Summary}

We summarize the modelling constrains and the related observations in Table 1 and show the simulated $408 \mathrm{MHz}$ total intensity, the $1.4 \mathrm{GHz}$ polarized intensity, $22.8 \mathrm{GHz}$ polarized intensity and polarization angle at $22.8 \mathrm{GHz}$ in Fig. 19 based on the ASS+RING disk field configuration. All maps shown have an angular resolution of $15^{\prime}$ and can be obtained on request from the authors.

\section{Discussion}

\subsection{Is a large halo field physically reasonable?}

Halo magnetic fields were detected just in a handful of nearby galaxies such as NGC 891 with a halo field running parallel to the plane or NGC 4631 showing a vertical field direction. The field strength is between $4 \mu \mathrm{G}$ and $6.5 \mu \mathrm{G}$ for $z$ between $2 \mathrm{kpc}$ to $5 \mathrm{kpc}$ (e.g. Dumke et al. 1995). However, the observed highlatitude RMs require a stronger halo field for the Galaxy as shown above.

In case the dynamo theory is relevant to describe the Galactic magnetic field evolution we learn that in a thick disk a asymmetric halo field can be generated as shown by Sokoloff \& Shukurov (1990). Simulations by Brandenburg et al. (1992) indicate that it takes a long time, which is comparable to the Hubble time, that the halo field reaches stability.

Considering hydrostatic equilibrium in the halo a mid-plane magnetic field strength of about $4 \mu \mathrm{G}$ with a scale height of $4.4 \mathrm{kpc}$ in the solar neighbourhood is needed (Kalberla \& Kerp 1998). The present halo model gives a field strength of about $7 \mu \mathrm{G}$ at $z$ of $1.5 \mathrm{kpc}$. This field sharply decreases towards the Galactic plane. Towards larger $z$ our model is insensitive to any scale height.

\subsection{The truncation of the $\mathrm{CR}$ disk electrons at $1 \mathrm{kpc}$}

$\mathrm{CR}$ electrons gyrate in a magnetic field with a radius $r_{\mathrm{g}} \approx$ $1 \times 10^{-6} E / B$ pc, where the energy $E$ is in units of $\mathrm{GeV}$ and the magnetic field strength is in $\mu \mathrm{G}$ (Ferrière 2001). Turbulence in the halo can be inferred from super-bubbles generating shockwaves from supernovae in OB clusters and stellar winds. The outer scale can be estimated to be about $100 \mathrm{pc}$, which is much larger than the gyrating radius $r_{\mathrm{g}}$. In that case diffusion of CRs both along and across magnetic field lines is strong. The diffusion along magnetic field lines is much larger when the turbulent component is smaller than the regular field component (Casse et al. 2001) as it is the case for the present halo model. Although the diffusion of CRs in a turbulent field is still debated (Ptuskin 2006), it seems unlikely that the CR electrons can be prevented to diffuse out of the Galactic plane into the region with the large halo field. Therefore the truncation of the CR electron density at $1 \mathrm{kpc}$ we need for our simulations seems unrealistic. 
Table 1. Observational features versus model constraints based on the NE2001 thermal electron model.

\begin{tabular}{ll}
\hline \hline Observational features & Model constrains \\
\hline $22.8 \mathrm{GHz}$ free-free emission template and $\beta_{22 / 408 \mathrm{MHz}}$ & NE2001 plus filling factor for thermal electrons \\
EGS RM profile along the Galactic plane & ASS+RING, ASS+ARM or BSS regular disk field, local field strength $2 \mu \mathrm{G}$ \\
High-latitude RMs asymmetry (to the plane and center) & Asymmetric toroidal halo field, field strength up to 10 $\mu \mathrm{G}$, CRs up to $1 \mathrm{kpc}$ \\
RM latitude profile at $100^{\circ}<l<120^{\circ}$ & Favour ASS+RING or ASS+ARM model \\
Longitude PI asymmetry to the centre at $22.8 \mathrm{GHz}$ & Favour ASS+RING or ASS+ARM model \\
Synchrotron emission longitude profiles: $408 \mathrm{MHz} \mathrm{I} / 22.8 \mathrm{GHz}$ PI & $\begin{array}{l}\text { Disk CR electron component and } 3 \mu \mathrm{G} \text { random field component } \\
\text { Isotropic high-latitude emission at } 408 \mathrm{MHz}\end{array}$ \\
$\begin{array}{l}\text { Local excess of the synchrotron emission } \\
\text { Strong depolarization at } 1.4 \mathrm{GHz}\end{array}$ & Large RM fluctuations along the line of sight \\
& by a small $n_{\mathrm{e}}$ filling factor and a coupling of the random field with $n_{\mathrm{e}}$ \\
\hline
\end{tabular}

However, if we give up the $1 \mathrm{kpc} C R$ electron truncation the modelled synchrotron emission will increase largely at high latitudes. As a consequence two ridges of strong emission running parallel to the Galactic plane will show up, which are not observed. In case we reduce the halo field to avoid the synchrotron emission problem, we are unable to model the RM data at high latitudes. To avoid this it is required to increase the thermal electron density or the scale height of the DIG.

\subsection{The scale height of the DIG}

The scale height of the DIG of about $1 \mathrm{kpc}$ results from fitting $D M \sin |b|$ versus $|z|$ for pulsars with independent distance measurements (Gómez et al. 2001; Cordes \& Lazio 2002). In fact the most stringent constraints come from distant pulsars located in globular clusters. However, there are just very few clusters with a large $z$ and the ISM has many voids and clumps, which may influence the scale height derived from limited data.

From the WHAM H $\alpha$ survey observations in the Perseus arm direction (Haffner et al. 1999; Reynolds et al. 1999) the $\mathrm{H} \alpha$ intensity can be fitted with a scale height of $1 \mathrm{kpc}$ and a mid-plane density of $0.06 \mathrm{~cm}^{-3}$. This electron density is larger than the mid-plane density of about $0.03 \mathrm{~cm}^{-3}$ in the NE2001 model. Since the NE2001 model fits the $D M \sin |b|-|z|$ relation very well, this might indicate that the electron density at high $z$ could be larger. The WHAM survey clearly shows WIM emission enhancements for large $|b|$ relative to the $E M \sin |b|$ distribution (Haffner et al. 1999; Reynolds et al. 1999), which indicates the presence of areas of large electron densities at high latitudes. However, how these high-latitude features are distributed is rather unclear at present.

In order to check how the high-latitude electron distribution influences the present simulations we run the HAMMURABI code by increasing the scale height of the DIG to $2 \mathrm{kpc}$ by keeping the mid-plane electron density from the NE2001 model unchanged. As a consequence from this model a regular halo field magnetic strength of about $2 \mu \mathrm{G}$ with a scale height of $4 \mathrm{kpc}$ is required, which sounds more reasonable compared to the $10 \mu \mathrm{G}$ magnetic field strength enforced by a $1 \mathrm{kpc}$ DIG scale height. In this case the halo field parameters agree with the estimates of Sokoloff \& Shukurov (1990) based on the dynamo theory. The $1 \mathrm{kpc}$ truncation of CR electron distribution could also be avoided that way. We conclude that the high-latitude thermal electron density is a critical issue for Galactic 3D-modelling and requires more investigations.

\section{Conclusions}

Galactic 3D-models of the distribution of thermal electrons, cosmic-ray electrons and magnetic fields were used to calculate via the HAMMURABI code all-sky total intensity, polarization and RM maps. A comparison with observed maps over a wide frequency range is made to adapt the $3 \mathrm{D}$-model parameter in the best possible, although still qualitative way (Table 1).

The main conclusions are:

- The NE2001 thermal electron model combined with a filling factor $f_{\mathrm{e}}$ as derived by Berkhuijsen et al. (2006) reproduces the optical thin $22.8 \mathrm{GHz}$ free-free emission template and also the observed spectral flattening in the Galactic plane between $408 \mathrm{MHz}$ and $22 \mathrm{MHz}$ caused by absorption of optically thick thermal gas. We find indications that the filling factor might be larger towards high $z$ than that found by Berkhuijsen et al. (2006).

- An ASS field configuration plus a reversal inside the solar circle either in a ring or between the inner edges of the Sagittarius-Carina arm and the Scutum-Crux arm is favoured by RMs of EGSs observed along the Galactic plane. A BSS field configuration also fits these RMs, however, it then fails to fit the observed RM gradient in latitude direction. The strength of the regular magnetic field component at the solar radius is $2 \mu \mathrm{G}$. The field in the Galactic centre region is constant at $2 \mu \mathrm{G}$ in a direction opposite to that at the solar radius.

- The strength of the Galactic random field component is $3 \mu \mathrm{G}$.

- A regular halo magnetic field with a maximum of $10 \mu \mathrm{G}$ at a height of $1.5 \mathrm{kpc}$ is required to account for the observed high-latitude RMs. This toroidal field is asymmetric to the Galactic plane and decreases sharply towards the plane. The variation of the field towards high $z$ is not a sensitive parameter in our simulation.

- The CR electron distribution has an exponential scale height of $1 \mathrm{kpc}$ and a radial scale length of $8 \mathrm{kpc}$. The disk is truncated at a height of $1 \mathrm{kpc}$. The local CR flux density at $10 \mathrm{GeV}$ is taken twice the directly measured value at earth. The energy power law spectral index $p$ is taken to be constant at -3 for frequencies above $408 \mathrm{MHz}$ and -2 below.

- The observed local excess of synchrotron emission is taken into account. The excess is either caused by an enhanced local CR electron density or by an increase of the local turbulent magnetic field. Our modelling can not distinguish between both possibilities.

- In addition to the filling factor we introduce a coupling factor between the thermal electron density and the random magnetic field component to reproduce the observed large RM fluctuations and the observed depolarization at $1.4 \mathrm{GHz}$.

However, a number of caveats remain to the above conclusions. In our simulation we use the NE2001 model as input for the thermal electron distribution and density. This requires a large halo field strength and hence a truncation of the CR electrons 
at $z=1 \mathrm{kpc}$. This might be not very realistic. Indeed, there are indications as discussed in Sect. 7.3 that the scale height of thermal electrons might be larger than $1 \mathrm{kpc}$. When the thermal electron scale height is increased by a factor of 2 we get a regular halo magnetic field of $2 \mu \mathrm{G}$ and the truncation of the CR electron density at $1 \mathrm{kpc}$ is avoided. We conclude that the high-latitude thermal electron density needs more investigations and thus the halo field parameters remain open.

For large areas along the Galactic plane RM data are still missing or very rare. A final answer on the magnetic field configuration depends on a much more complete RM data set. Pulsar RM data are not yet included in our model, although they are potentially better tracers of the regular Galactic magnetic field along the line of sight than those from EGSs as they show no intrinsic RM. Pulsar RMs are clearly important to detail the disk magnetic field throughout the Galaxy on smaller scales than in our model and might also reveal deviations from a general regular pattern being assumed here. Despite of significant observational progress over the last years (e.g. Han et al. 2006) more RM data are clearly needed to be conclusive and pulsar distances need to be better constrained in addition.

We note that our 3D-model differs from previous models, which were derived from selected observational data. These models then fail to properly reproduce other observations, which, however, trace relevant information of the interstellar medium, which can not be ignored on the way towards a physical relevant model.

Acknowledgements. X.H. Sun acknowledges support by the European Community Framework Programme 6, Square Kilometre Design Study (SKADS), contract no 011938. We like to thank JinLin Han for providing RM data prior to publication used to create the synthesized RM map shown in Fig. 3 and used in Fig. 12. We also thank Jo-Anne Brown for providing her unpublished RM data from the extended CGPS included in Fig. 12. We acknowledge discussions with Elly Berkhuijsen and Rainer Beck. We thank Rainer Beck and Patricia Reich for careful reading of the manuscript and in particular the anonymous referee for valuable comments.

\section{References}

Barwick, S. W., Beatty, J. J., \& Bower, C. R., et al. 1998, ApJ, 498, 779

Beck, R. 2001, Space Sci. Rev., 99, 243

Beck, R., \& Krause, M. 2005, AN, 326, 414

Beck, R., Bandenburg, A., Moss, D., Shukurov, A., \& Sokoloff, D. 1996, ARA\&A, 34155

Berkhuijsen, E. M. 1973, A\&A, 24, 143

Berkhuijsen, E. M., Haslam, C. G. T. \& Salter, C. J. 1971, A\&A, 14, 252

Berkhuijsen, E. M., Mitra, D., \& Müller, P. 2006, AN, 327, 82

Beuermann, K., Kanbach, G., \& Berkhuijsen, E. M. 1985, A\&A, 153, 17

Brandenburg, A., Donner, K. J., Moss, D., et al. 1992, A\&A, 259, 453

Breitschwerdt, D., \& de Avillez, M. A. 2006, A\&A, 452, L1

Broadbent, A., Haslam, C. G. T., \& Osborne, J. L. 1989, MNRAS, 237, 381

Brown, J. C., Taylor, A. R., \& Jackel, B. J. 2003a, ApJS, 145, 213

Brown, J. C., Taylor, A. R., Wielebinski, R., \& Müller, P. 2003b, ApJ, 592, L29

Brown, J. C., Haverkorn, M., Gaensler, B. M., et al. 2007, ApJ, 663, 258

Brouw, W. N., \& Spoelstra, T. A. T. 1976, A\&AS, 26, 129

Burn, B. J. 1966, MNRAS, 133, 67

Casse, F., Lemoine, M., \& Pelletier, G. 2001, Phys. Rev. D, 65, 023002

Cordes, J. M., \& Lazio, T. J. W. 2002, [arXiv: astro-ph/0207156]

Cordes, J. M., \& Lazio, T. J. W. 2003, [arXiv: astro-ph/0301598]

Cordes, J. M., Weisberg, J. M., Frail, D. A., Spangler, S. R., \& Ryan, M. 1991, Nature, 354, 121

Davies, R. D., Dickinson, C., Banday, A. J., et al. 2006, MNRAS, 370, 1125

Dennison, B., Simonetti, J. H., \& Topasna, G. A. 1998, Publ. Astron. Soc. Australia, 15, 147

Dickinson, C., Davies, R. D., \& Davis, R. J. 2003, MNRAS, 341, 369

Dineen, P., \& Coles, P. 2005, MNRAS, 362, 403

Dumke, M., Krause, M., Wielebinski, R., \& Klein, U. 1995, A\&A, 302, 691

Ferrière, K. M. 2001, Rev. Mod. Phys., 73, 1031

Finkbeiner, D. P. 2003, ApJS, 146, 407
Fleishman, G. D., \& Tokarev, Yu. V. 1995, A\&A, 293, 565

Fuchs, B., Breitschwerdt, D., de Avillez, M. A., Dettbarn, C., \& Flynn, C. 2006, MNRAS, 373, 993

Gaensler, B. M., Dickey, J. M., McClure-Griffiths, N. M., et al. 2001, ApJ, 549, 959

Gaensler, B. M., Beck, R., \& Ferreti, L. 2004, in Science with the Square Kilometre Array, ed. C. Carilli, \& S. Rawlings, New Astron. Rev., 48, 1003 Gaustad, J. E., McCullough, P. R., Rosing, W., \& van Buren, D. 2001, PASP, 113,1326

Gómez, G. C., Benjamin, R. A., \& Cox, D. P. 2001, AJ, 122, 908

Górski, K. M., Hivon, E., Banday, A. J., et al. 2005, ApJ, 622, 759

Grimani, C., Stephens, S. A., \& Cafagna, F. S., et al. 2002, A\&A, 392, 287

Haffner, L. M., Reynolds, R. J., \& Tufte, S. L. 1999, ApJ, 523, 223

Haffner, L. M., Reynolds, R. J., Tufte, et al. 2003, ApJS, 149, 405

Han, J. L., \& Qiao, G. J. 1994, A\&A, 288, 759

Han, J. L., \& Wielebinski, R. 2002, ChJAA, 2, 293

Han, J. L., Manchester, R. N., Berkhuijsen, E. M., \& Beck, R. 1997, A\&A, 322, 98

Han, J. L., Manchester, R. N., \& Qiao, G. J. 1999, MNRAS, 306, 371

Han, J. L., Ferrière, K., \& Manchester, R. N. 2004, ApJ, 610, 820

Han, J. L., Manchester, R. N., Lyne, A. G., Qiao, G. J., \& van Straten, W. 2006, ApJ, 642, 868

Haslam, C. G. T., Salter, C. J., Stoffel, H., \& Wilson, W. E. 1982, A\&AS, 47, 1

Haverkorn, M., Gaensler, B. M., Brown, J. C., et al. 2006, ApJ, 637, L33

Hill, A. S., Reynolds, R. J., Benjamin, R. A., \& Haffner, L. M. 2007, in SINS Small Ionized and Neutral Structures in the Diffuse Interstellar Medium, ed. M. Haverkorn, \& W. M. Goss, ASP, 250

Hinshaw, G., Nolta, M. R., Bennett, C. L., et al. 2007, ApJS, 170, 288

Johnston-Hollitt, M., Hollitt, C. P., \& Ekers, R. D. 2004, in The Magnetized Interstellar Medium, ed. B. Uyanıker, W. Reich, \& R. Wielebinski, Copernicus $\mathrm{GmbH}, 13$

Kalberla, P. M. W., \& Kerp, J. 1998, A\&A, 339, 745

Kobayashi, T., Komori, Y., Yoshida, K., \& Nishimura, J. 2004, ApJ, 601, 340

Kogut, A., Dunkley, J., Bennett, C. L., et al. 2007, ApJ, 665, 355

Law, C. J. 2007, Ph.D., Northwestern University, Evanston, Illinois

Madsen, G. J., Reynolds, R. J., \& Haffner, L. M. 2006, ApJ, 652, 401

Martel, H. 2005, Technical Report UL-CRC/CTN-RT003

[arXiv: astro-ph/0506540]

Minter, A. H., \& Spangler, S. R. 1996, ApJ, 458, 194

Mitra, D., Wielebinski, R., Kramer, M., \& Jessner, A. 2003, A\&A, 398, 993

Nord, M. E., Henning, P. A., Rand, R. J., Lazio, T. J. W., \& Kassim, N. E. 2006, AJ, 132, 242

Page, L., Hinshaw, G., Komatsu, E., et al. 2007, ApJS, 170, 335

Paladini, R., Davies, R. D., \& DeZotti, G. 2004, MNRAS, 347, 237

Paladini, R., DeZotti, G., Davies, R. D., \& Giard, M. 2005, MNRAS, 360, 1545

Peterson, J. D., \& Webber, W. R. 2002, ApJ, 575, 217

Phillipps, S., Kearsey, S., Osborne, J. L., Haslam, C. G. T., \& Stoffel, H. 1981a, A\&A, 98, 286

Phillipps, S., Kearsey, S., Osborne, J. L., Haslam, C. G. T., \& Stoffel, H. 1981b, A\&A, 103, 405

Pohl, M., \& Esposito, J. A. 1998, ApJ, 507, 327

Prouza, M., \& Șmída, R. 2003, A\&A, 410, 1

Ptuskin, V. 2006, J. Phys., Conf. Ser., 47, 113

Quireza, C., Rood, R. T., Bania, T. M., Balser, D. S., \& Maciel, W. J. 2006, ApJ, 653,1226

Rand, R. J., \& Kulkarni, S. R. 1989, ApJ, 343, 760

Reich, P. 2003, Acta Astron. Sin., 44, Suppl., 130

Reich, P., \& Reich, W. 1986, A\&AS, 63, 205

Reich, P., \& Reich, W. 1988a, A\&AS, 74, 7

Reich, P., \& Reich, W. 1988b, A\&A, 196, 211

Reich, P., Testori, J. C., \& Reich, W. 2001, A\&A, 376, 861

Reich, P., Reich, W., \& Testori, J. C. 2004, in The Magnetized Interstellar Medium, ed. B. Uyanıker, W. Reich, \& R. Wielebinski, Copernicus GmbH, 63

Reich, W. 1982, A\&AS, 48, 219

Reich, W. 2006, in Cosmic Polarization, ed. R. Fabbri, Research Signpost, 91

Reich, W., Fürst, E., Reich, P., et al. 2004, in The Magnetized Interstellar Medium, ed. B. Uyanıker, W. Reich, \& R. Wielebinski, Copernicus GmbH, 45

Reynolds, R. J., Haffner, L. M., \& Tufte, S. L. 1999, ApJ, 525, L21

Roger, R. S., Costain, C. H., Landecker, T. L., \& Swerdlyk, C. M. 1999, A\&AS, 137, 7

Rohlfs, K., \& Wilson, T. L. 2000, Tools of Radio Astronomy, third revised and enlarged edition (Springer)

Roy, S., Rao, A. P., \& Subrahmanyan, R. 2005, MNRAS, 360, 1305

Rybicki, G. B., \& Lightman, A. P. 1979, Radiative processes in astrophysics (New York: Wiley-interscience)

Schlegel, D. J., Finkbeiner, D. P., \& Davis, M. 1998, ApJ, 500, 525 
Schraml, J., \& Mezger, P. G. 1969, ApJ, 156, 269

Sokoloff, D., \& Shukurov, A. 1990, Nature, 347, 51

Sokoloff, D. D., Bykov, A. A., Shukurov, A., et al. 1998, MNRAS, 299, 189

Stanev, T. 1997, ApJ, 479, 290

Strong, A. W., \& Moskalenko, I. V. 1998, ApJ, 509, 212

Strong, A. W., Moskalenko, I. V., \& Reimer, O. 2000, ApJ, 537, 763

Strong, A. W., Moskalenko, I. V., \& Reimer, O. 2004, ApJ, 613, 962

Sun, X. H., \& Han, J. L. 2004, in The Magnetized Interstellar Medium, ed. B.

Uyanıker, W. Reich, \& R. Wielebinski (Copernicus GmbH), 25

Sun, X. H., Han, J. L., Reich, W., et al. 2007, A\&A, 463, 993

Taylor, A. R., Gibson, S. J., Peracaula, M., et al. 2003, AJ, 125, 3145

Taylor, J. H., \& Cordes, J. M. 1993, ApJ, 411, 674

Testori, J. C., Reich, P., \& Reich, W. 2004, in The Magnetized Interstellar Medium, ed. B. Uyanıker, W. Reich, \& R. Wielebinski (Copernicus GmbH), 57
Tinyakov, P. G., \& Tkachev, I. I. 2002, Astropart. Phys., 18, 165

Uyanıker, B., Fürst, E., Reich, W., Reich, P., \& Wielebinski, R. 1998, A\&AS, 132,401

Waelkens, A. 2005, Diploma Thesis, Ludwig-Maximilian-Universität München Webber, W. R., Simpson, G. A., \& Cane, H. V. 1980, ApJ, 236, 448

Westerhout, G., Seeger, C. L., Brouw, W. N., \& Tinbergen, J. 1962, Bull. Astron. Inst. Netherlands, 16, 187

Widrow, L. M. 2002, Rev. Mod. Phys., 74, 775

Wielebinski, R., Shakeshaft, J. R., \& Pauliny-Toth, I. I. K. 1962, The Observatory, 82, 158

Wilkinson, A., \& Smith, F. G. 1974, MNRAS, 167, 593

Wolleben, M. 2007, ApJ, 664, 349

Wolleben, M., \& Reich, W. 2004, A\&A, 427, 537

Wolleben, M., Landecker, T. L., Reich, W., \& Wielebinski, R. 2006, A\&A, 448, 441 